Article

\title{
'One-pot' Synthesis of Dihydrobenzo[4,5][1,3]oxazino[2,3-a] isoquinolines via a Silver(I)-Catalyzed Cascade Approach
}

\author{
Baifeng Jiang ${ }^{1}$, Yu Zhou ${ }^{2, *}$, Qingya Kong ${ }^{1}$, Hualiang Jiang ${ }^{1,2}$, Hong Liu ${ }^{2}$ and Jian Li ${ }^{1, *}$
}

1 Shanghai Key Laboratory of New Drug Design, School of Pharmacy, East China University of Science and Technology, 130 Mei Long Road, Shanghai 200237, China

2

State Key Laboratory of Drug Research, Shanghai Institute of Materia Medica, Chinese Academy of Sciences, 555 Zu Chong Zhi Road, Shanghai 201203, China

* Authors to whom correspondence should be addressed; E-Mails: zhouyu@mail.shcnc.ac.cn (Y.Z.); jianli@ecust.edu.cn (J.L.); Tel./Fax: +86-21-5080-7042 (Y.Z.); +86-21-6425-2584 (J.L.).

Received: 26 November 2012; in revised form: 4 January 2013 / Accepted: 5 January 2013 / Published: 11 January 2013

\begin{abstract}
An efficient approach for the synthesis of biologically interesting fused tetracyclic isoquinolines in high yields and with a broad substrate scope has been developed. The strategy features an $\mathrm{AgNO}_{3}$ catalyzed 'one-pot' cascade process involving formation of two new $\mathrm{C}-\mathrm{N}$ bonds and one new $\mathrm{C}-\mathrm{O}$ bond.
\end{abstract}

Keywords: silver; one-pot; cascade transformation; isoquinolines; heterocyclic molecule

\section{Introduction}

Fused isoquinolines are widely distributed in alkaloids and biologically important synthetic substances. They exhibit a broad spectrum of biological properties, such as antitumor activity [1,2], TC-PTP and PTP1B inhibitory activities [3], and HIV inhibitory potencies [4,5]. Therefore, the development of new synthetic strategies for the efficient preparation of fused isoquinolines is in considerable demand. Compared to the stepwise approach, cascade reactions represent an attractive strategy in synthesis because multiple bond-forming and -cleaving events can be combined into a single reaction operation. Recently, transition-metal-catalyzed cascade processes have received significant attention [6-19]. Among these transition metals, silver salts have long been believed to have low catalytic efficiency, and most commonly served as either co-catalysts or weak Lewis acids [20]. 
However, in recent years they have been extensively employed to activate alkyne, alkene, and allene functionalities under mild conditions and at low catalyst loadings [21-34].

In our ongoing efforts for the development of the efficient synthetic methods for the construction of potential bioactive fused polycyclic compounds through exploration of new catalytic cascade strategies [35-41], we envisioned that the direct assembly of nitrogen-containing tetracyclic isoquinoline structures 3 could be realized in a 'one-pot' operation from readily available and functional 2-substituted-ethynyl benzaldehydes 1 with 2-aminoarylmethanols 2 (Scheme 1). In this design, the condensation of the starting materials $\mathbf{1}$ and $\mathbf{2}$ would be primed for the formation of the Ag-complex (B or $\mathbf{B}^{\prime}$ ) in the presence of Ag salts as catalyst. The resulting species would be subject to a cascade nucleophilic attack to give the target scaffold $\mathbf{3}$. Successful execution of the proposal would lead to highly functionalized isoquinolines, which are particularly attractive for further elaboration in diversity oriented synthesis. Recently, some progress has been made via tandem nucleophilic addition and cyclization to give fused tetracyclic isoquinolines by using $o$-alkynylbenzaldehyde as the starting material in the presence of various Lewis acid catalysts, such as $\mathrm{AuCl}$ [17], $\mathrm{In}(\mathrm{OTf})_{3}$ [18], $\mathrm{Yb}(\mathrm{OTf})_{3}$ [13], $\mathrm{CuI}$ [16], $\mathrm{AgNO}_{3}$ [28,29], and $\mathrm{Ph}_{3} \mathrm{PAuMe} /$ chiral Brønsted acids [15]. However, to the best of our knowledge, there is no report involving the synthesis of dihydrobenzo[4,5][1,3]oxazino[2,3-a] isoquinolines 3 via a $\mathrm{AgNO}_{3}$-catalyzed one-pot domino process. Herein, we wish to disclose our recent results in this area.

Scheme 1. A plausible mechanism.

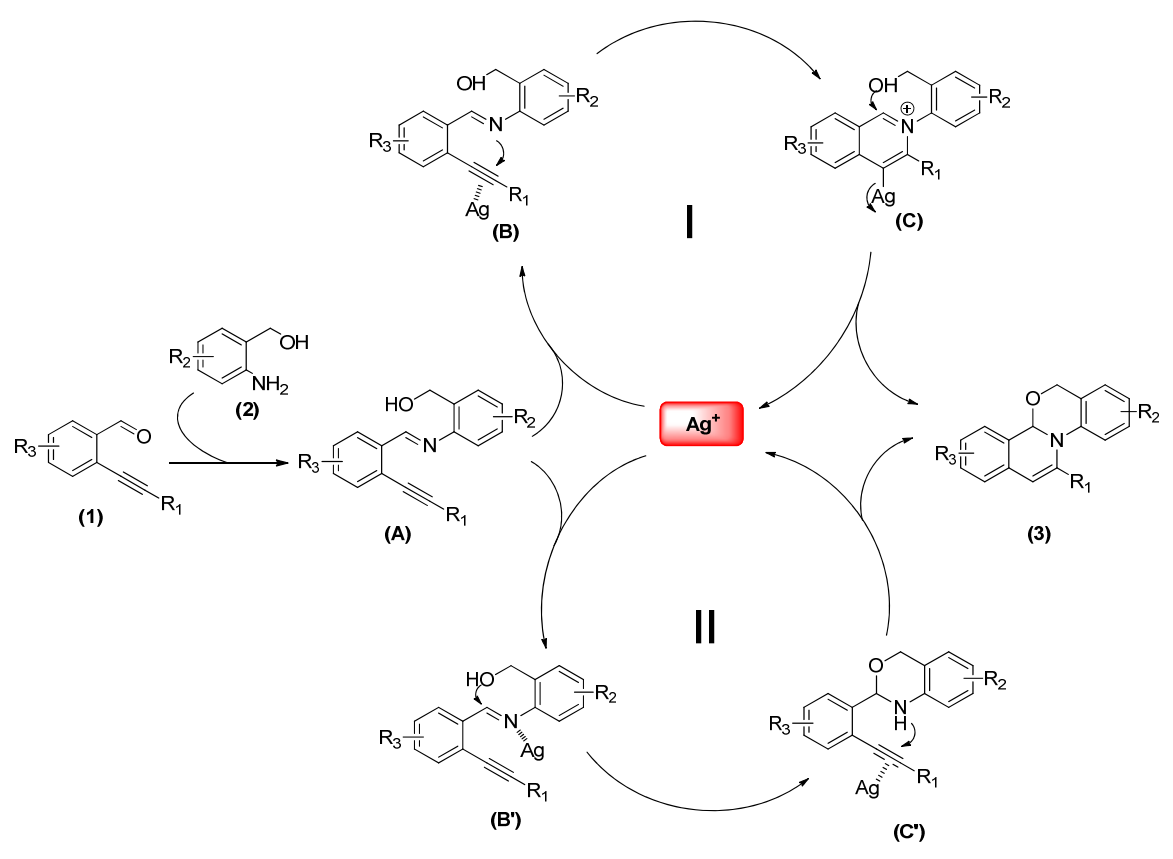

\section{Results and Discussion}

To fulfill the hypothesis, we carried out the experiments using 2-(phenylethynyl)benzaldehyde (1a) and (2-aminophenyl)methanol (2a) as model substrates (as shown in Table 1). In our previous studies, we found that some gold-complex and/or silver salts were highly efficient catalysts for cascade reactions involving the activation of alkynyl groups [35-41]. Therefore, several gold catalysts, including $\mathrm{AuCl}\left(\mathrm{PPh}_{3}\right), \quad \mathrm{AuCl}, \quad$ (acetonitrile)[(2-biphenyl)di-tert-butylphosphine]gold(I) hexafluoro-antimonate 
( $\mathrm{Au}$ catalyst I), and 2-dicyclohexylphosphino-2',4',6'-triisopropyl-biphenyl gold(I) bis(trifluoromethanesulfonyl)imide (Au catalyst II) were firstly investigated in a sealed tube using dry toluene as the solvent at $100{ }^{\circ} \mathrm{C}$ for $3 \mathrm{~h}$. Disappointingly, the desirable cascade products were obtained in only $28 \%-75 \%$ yields (Table 1, entries 1-4). Different silver salts, such as $\mathrm{AgOOCCF}_{3}, \mathrm{AgNO}_{3}$, $\mathrm{AgBF}_{4}$ and AgOTf, were also subsequently screened, and $\mathrm{AgNO}_{3}$ was proved to be the most effective one for this transformation (Table 1, entries 5-8), and the product 3Aa could be obtained in 93\% yield (Table 1, entry 6). It is apparent that the solvent has a significant influence on the yield of this reaction (Table 1, entries 9-14). Toluene was found to be the optimal solvent for this transformation (Table 1, entries 6, 9-14), although when $\mathrm{DMSO}, \mathrm{CH}_{3} \mathrm{CN}$ and 1,4-dioxane were used instead of toluene, the desired product 3Aa can also obtained with good yields (Table 1, entries 12-14). The yield of the product has no significant difference when we decrease the reaction temperature to $80{ }^{\circ} \mathrm{C}$ in toluene (Table 1, entry 15). However, when DMSO, $\mathrm{CH}_{3} \mathrm{CN}$ and 1,4-dioxane were used, decreasing temperature appears to have a negative impact on the yield of product (Table 1, entries 16-18). We further decreased reaction temperature to $50{ }^{\circ} \mathrm{C}$ and room temperature, but these changes adversely affected the product yield, and product 3Aa was obtained in only $65 \%$ and $30 \%$ yield, respectively (Table 1, entries 19-20).

Table 1. Optimization of the reaction conditions ${ }^{\mathrm{a}}$.<smiles>O=Cc1ccccc1C#Cc1ccccc1</smiles>

$1 \mathrm{~A}$

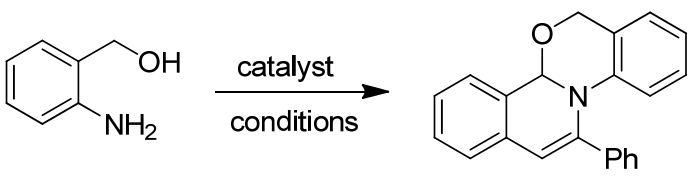

$2 \mathbf{a}$
3Aa

\begin{tabular}{lllll}
\hline Entry & Catalyst system $^{\boldsymbol{b}}$ & Solvent & Temp $\left({ }^{\circ} \mathbf{C}\right)$ & Yield (\%) \\
\hline 1 & $\mathrm{AuCl}\left(\mathrm{PPh}_{3}\right)$ & Toluene & 100 & 75 \\
2 & $\mathrm{AuCl}$ & Toluene & 100 & 49 \\
3 & $\mathrm{Au}$ catalyst I & Toluene & 100 & 58 \\
4 & $\mathrm{Au} \mathrm{catalyst} \mathrm{II}$ & Toluene & 100 & 28 \\
5 & $\mathrm{AgOOCCF}_{3}$ & Toluene & 100 & 60 \\
$\mathbf{6}$ & $\mathbf{A g N O}_{3}$ & Toluene & $\mathbf{1 0 0}$ & $\mathbf{9 3}$ \\
7 & $\mathrm{AgBF}_{4}$ & Toluene & 100 & 57 \\
8 & $\mathrm{AgOTf}$ & Toluene & 100 & 92 \\
9 & $\mathrm{AgNO}_{3}$ & MeOH & reflux & 77 \\
10 & $\mathrm{AgNO}_{3}$ & THF & reflux & 60 \\
11 & $\mathrm{AgNO}_{3}$ & DMF & 100 & 71 \\
12 & $\mathrm{AgNO}_{3}$ & DMSO & 100 & 92 \\
13 & $\mathrm{AgNO}_{3}$ & CHCN & reflux & 87 \\
14 & $\mathrm{AgNO}_{3}$ & Dioxane & 100 & 83 \\
$\mathbf{1 5}$ & $\mathbf{A g N O}_{3}$ & Toluene & $\mathbf{8 0}$ & $\mathbf{9 4}$ \\
16 & $\mathrm{AgNO}_{3}$ & DMSO & 80 & 75 \\
17 & $\mathrm{AgNO}_{3}$ & CH & 78 \\
18 & $\mathrm{AgNO}_{3}$ & Dioxane & 80 & 77 \\
19 & $\mathrm{AgNO}_{3}$ & Toluene & 80 & 65 \\
20 & $\mathrm{AgNO}_{3}$ & Toluene & RT & 30 \\
$\mathbf{2 1}$ & $\mathbf{A g N O}_{3}$ & Toluene & $\mathbf{8 0}$ & $\mathbf{9 4}$ \\
\hline
\end{tabular}

${ }^{a} \mathbf{1 A}(0.1 \mathrm{mmol}), \mathbf{2 a}(0.2 \mathrm{mmol})$ and catalyst $(5 \mathrm{~mol} \%)$ in the specified solvent $(2 \mathrm{~mL})$ were reacted in a sealed vial; ${ }^{\mathrm{b}}$ Au catalyst I $=($ acetonitrile) $[(2$-biphenyl)di-tert-butylphosphine]gold (I) hexafluoroantimonate; $\mathrm{Au}$ catalyst II = 2-dicyclohexylphosphino-2',4',6'-triisopropyl-biphenyl gold(I) bis(trifluoromethane-sulfonyl)imide; ${ }^{\mathrm{c}} 1.2$ eqv. of $2 \mathrm{a}$ was used. 
Furthermore, the amount of starting material 2a was also screened, and the results demonstrated that the same excellent yield for desired product $3 \mathrm{Aa}$ were observed in toluene at $80{ }^{\circ} \mathrm{C}$ for $3 \mathrm{~h}$ when 1.2 equiv. of 2 a was used (Table 1, entry 21).

To explore the scope and limitation of this cascade reaction, we surveyed the diversity of the starting materials by the structural variations of both 2-substituted-ethynyl benzaldehydes $(\mathbf{1} \mathbf{A}-\mathbf{H})$ and 2-aminoarylmethanols $(\mathbf{2} \mathbf{a}-\mathbf{j})$. As shown in Table 2, notably, the corresponding fused tetracyclic isoquinolines products $\mathbf{3 A a}-\mathbf{B j}$ were efficiently produced in moderate to excellent yields (45\%-94\%). The nature of the 2-aminoarylmethanol and the substituents attached to the triple bond of benzaldehydes has a major impact on the yield of the transformation. When 2-phenylethynylbenzaldehyde (1A) was treated with different substituted 2-aminoarylmethanols $2 \mathbf{a}-\mathbf{e}$, excellent yields were achieved (Table 2, entries 1-5). Moreover, when introducing substituent groups such as fluoro, chloro, or methyl groups onto the phenyl ring in the 2-phenylethynylbenzaldehydes, most of the desired products were obtained with high yields (Table 2, entries 6-22). Nevertheless, some substituted 2-aminoarylmethanols with bromo or methyl groups (Table 2, entries 8-10, 17-18 and 22), especially 6-methyl-2-aminophenylmethanol (Table 2, entry 10), result in a decrease in the yield of the target products, presumably due to the influence of electronic and/or steric effects. Subsequently, we prepared substrates $\mathbf{1 F}$ and $\mathbf{1 G}$ by introducing methyl and fluoro groups in the 4-position and 5-postion of 2-phenylethynylbenzaldehyde (1A), respectively. Results of further investigations demonstrated that all tested substrates were tolerated in this cascade transformation, giving good to excellent yields (Table 2, entries 23-30). However, a relatively lower 55\% yield of product 3Gh was obtained in the reaction of 2-phenylethynyl-4-methylbenzaldehyde (1G) with 3-aminonaphthalenylmethanol (2h). We speculate that the electronic effect of naphthalene ring was responsible for the decrease in the yield. Furthermore, an alkyl ( $n$-hexyl) group at the $\mathrm{R}^{1}$ position was also tolerated, and excellent yields were obtained (Table 2, entries 31-32). Finally, a $N$-containing heterocyclic substrate (2-aminopyridinylmethanol, $\mathbf{2} \mathbf{j}$ ) was investigated in this cascade transformation, but only moderate yields were obtained (Table 2, entries 33-34). In view of these findings, this cascade strategy could serve as a general approach for the preparation of fused tetracyclic isoquinoline complex molecular architectures.

Table 2. Silver-mediated one-pot domino synthesis of target compounds $\mathbf{3}^{\text {a }}$.

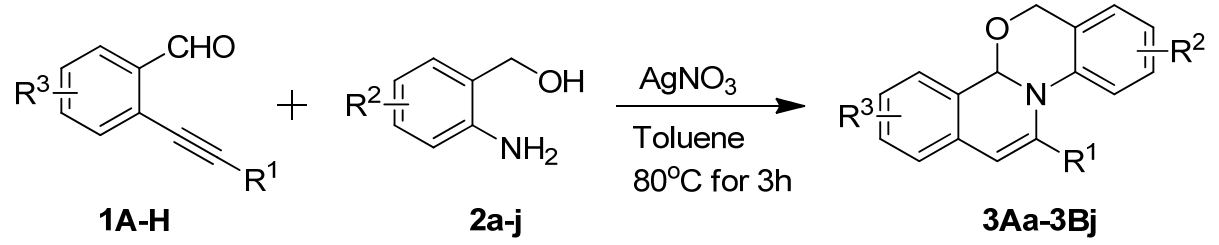

Entry Yield (\%)


Table 2. Cont.

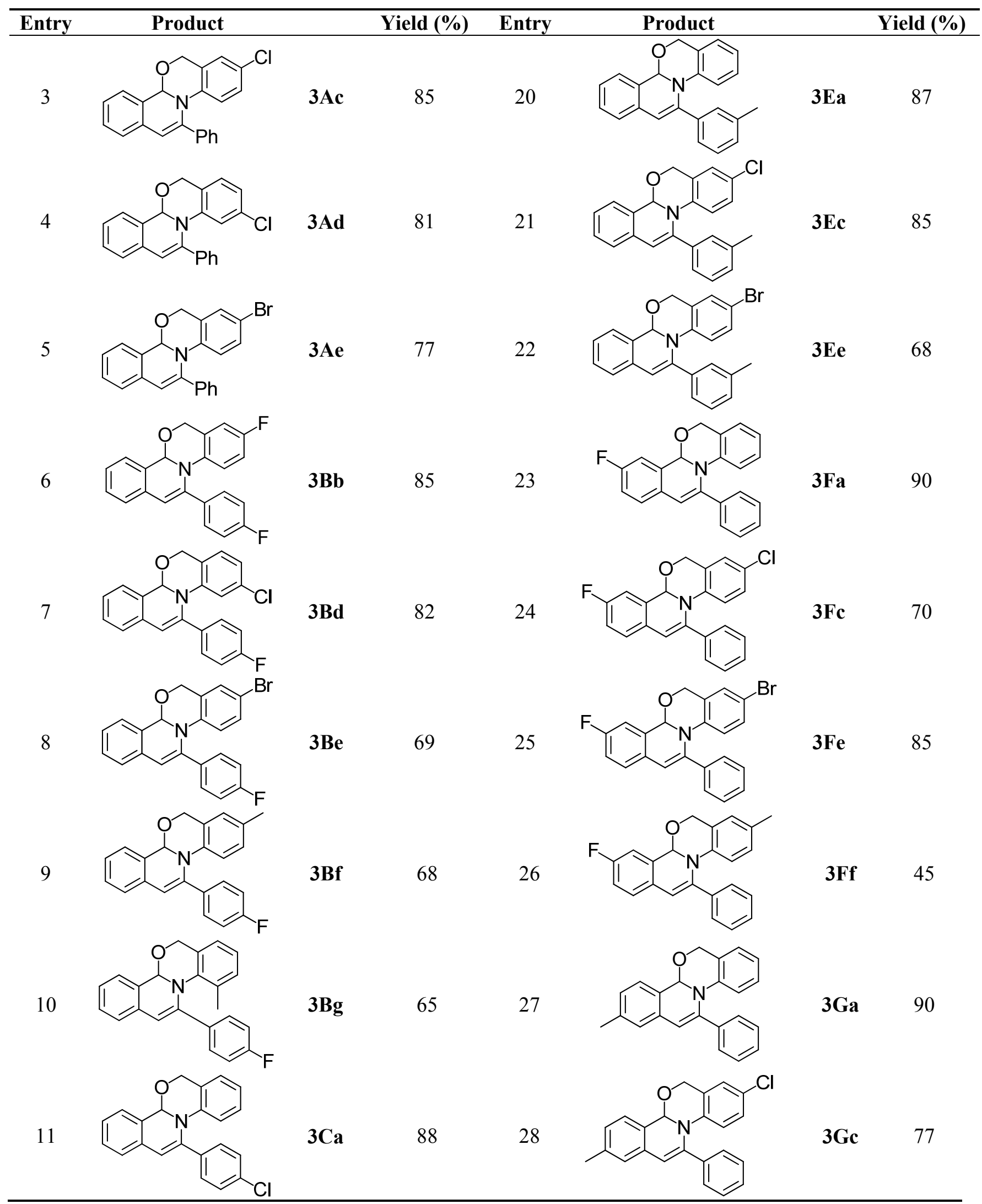


Table 2. Cont.

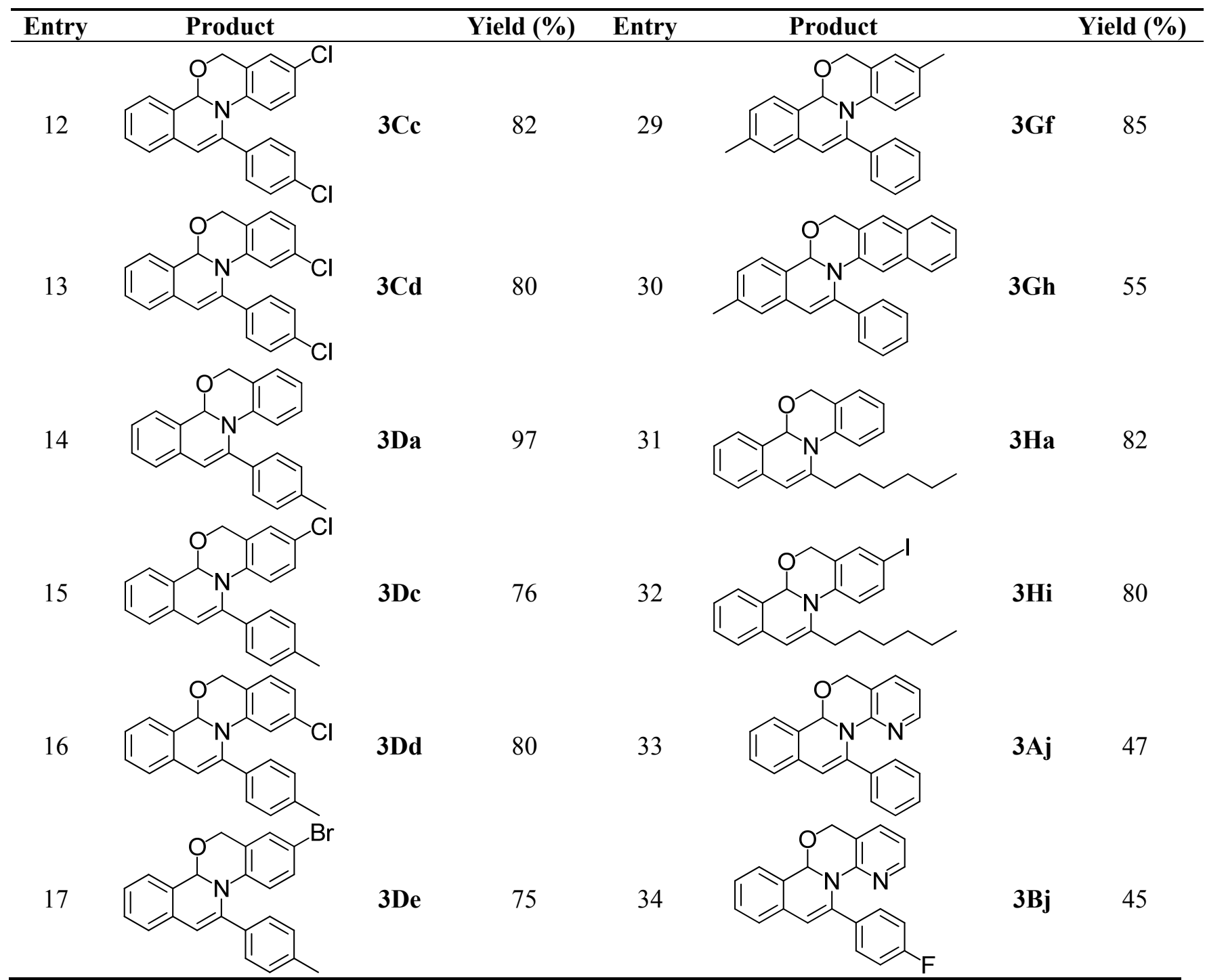

${ }^{\mathrm{a}} \mathbf{1 A}(0.1 \mathrm{mmol}), \mathbf{2 a}(0.12 \mathrm{mmol})$ and catalyst $(5 \mathrm{~mol} \%)$ in toluene $(2 \mathrm{~mL})$ were perfomed in a sealed vial.

Scheme 1 depicts a plausible mechanism for this cascade transformation. The condensation of the starting materials $\mathbf{1}$ and $\mathbf{2}$ generates the key imine intermediate $\mathbf{A}$, which subsequently can be converted into the final product 3 via two possible catalytic pathways (I and II). In pathway I [17,42], the imine intermediate $\mathbf{A}$ is activated by $\mathrm{AgNO}_{3}$ to form the $\pi$-Ag complex $\mathbf{B}$, and futher generates the $\mathrm{N}$-aryl imine cation $\mathbf{C}$ through an intramolecular nucleophilic addition, which is subject to a subsequent nucleophilic attack and proton transformation to afford the target scaffold 3 . In the conceivable alternative pathway II $[17,28]$, the aminal intermediate $\mathbf{C}^{\prime}$ is probably formed by an intramolecular nucleophilic addition of iminoalkyne Ag-complex B'. Then, the Ag-mediated intramolecular hydroamination reaction of $\mathbf{C}^{\prime}$ and subsequent protonation results in the formation of the target product 3. The target product 3Aa was further characterized by X-ray crystallography (Figure 1, see Supporting Information for details). 
Figure 1. X-ray crystallographic structure of 3Aa.

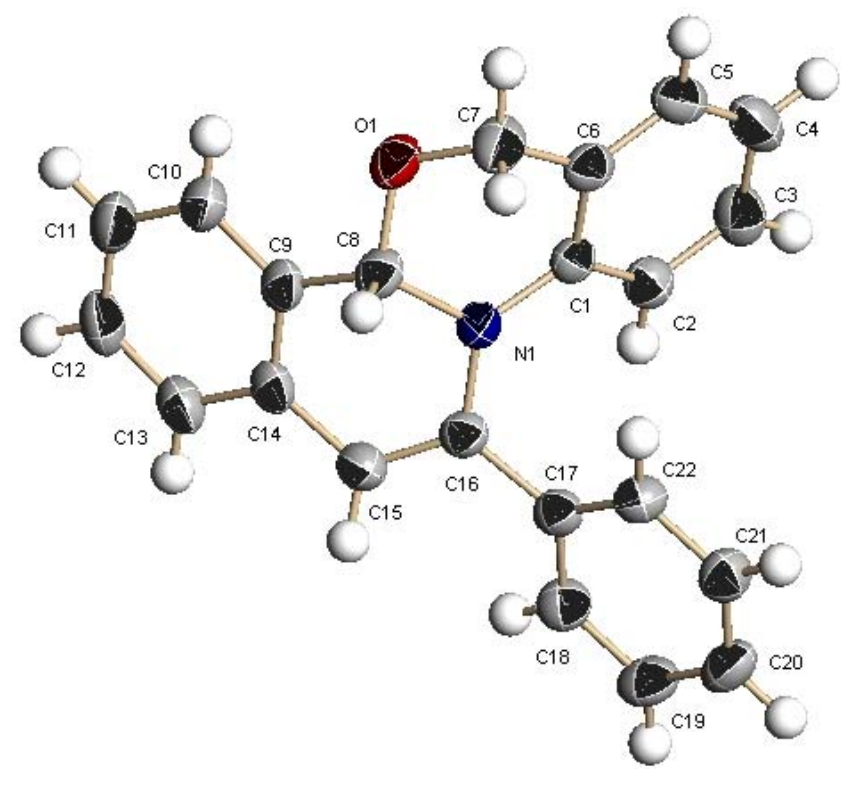

\section{Experimental}

\subsection{General}

2-Aminoarylmethanols $\mathbf{2 a - j}$ and the two 2-substituted-ethynyl benzaldehydes $\mathbf{1 A}$ and $\mathbf{2 B}$ are commercially available starting materials. The five remaining 2-substituted-ethynyl benzaldehydes $\mathbf{1 C}-\mathbf{H}$ were prepared as indicated in the following methods. Commercially available reagents and solvents were used without further purification. Column chromatography was carried out on silica gel. ${ }^{1} \mathrm{H}$ and ${ }^{13} \mathrm{C}-\mathrm{NMR}$ spectra were obtained on Varian Mercury-300, Varian Mercury-400 and Varian Mercury-500 spectrometers (TMS as IS). Chemical shifts were reported in parts per million (ppm, $\delta$ ) downfield from tetramethylsilane. Proton coupling patterns are described as singlet (s), doublet (d), triplet $(\mathrm{t})$, quartet $(\mathrm{q})$, multipet $(\mathrm{m})$ and broad (br). Low- and high-resolution mass spectra (LRMS and HRMS) were measured on a Finnigan MAT 95 spectrometer.

\subsection{General Procedure for Synthesis of 2-Substituted-ethynyl Benzaldehyde Derivatives $\mathbf{1 C}-\mathbf{H}$ (1C as an Example)}

To a solution of 2-bromobenzaldehyde $(0.37 \mathrm{~g}), \mathrm{PdCl}_{2}\left(\mathrm{PPh}_{3}\right)_{2}(28 \mathrm{mg})$, and $\mathrm{CuI}(3.8 \mathrm{mg})$ in of $\mathrm{Et}_{3} \mathrm{~N}$ $(20 \mathrm{~mL})$ was added phenylacetylene $(0.2 \mathrm{~g})$. The resulting mixture was heated under a nitrogen atmosphere at $60{ }^{\circ} \mathrm{C}$ for $4 \mathrm{~h}$. After the reaction was completed, the reaction mixture was concentrated under reduced pressure, and the resulting residue was purified by flash column chromatography $(\mathrm{PE} / \mathrm{EA}=50 / 1, \mathrm{v} / \mathrm{v}$, as an eluent) to give 2-((4-chlorophenyl)ethynyl)benzaldehyde (1C) in 90\% yield. ${ }^{1} \mathrm{H}-\mathrm{NMR}\left(500 \mathrm{MHz}, \mathrm{CDCl}_{3}\right) \delta 10.61(\mathrm{~s}, 1 \mathrm{H}), 7.94(\mathrm{~d}, J=7.8 \mathrm{~Hz}, 1 \mathrm{H}), 7.63(\mathrm{~d}, J=7.6 \mathrm{~Hz}, 1 \mathrm{H}), 7.58$ $(\mathrm{t}, J=7.5 \mathrm{~Hz}, 1 \mathrm{H}), 7.47(\mathrm{~m}, 3 \mathrm{H}), 7.35$ (d, $J=8.4 \mathrm{~Hz}, 2 \mathrm{H}) .{ }^{13} \mathrm{C}-\mathrm{NMR}\left(125 \mathrm{MHz}, \mathrm{CDCl}_{3}\right) \delta 191.47$, 135.84, 135.19, 133.84, 133.25, 132.91, 128.93, 128.86, 127.46, 126.41, 120.83, 95.09, 85.91. LRMS (ESI) $m / z 241[\mathrm{M}+\mathrm{H}]^{+}$; HRMS (ESI) $m / z$ calcd $\mathrm{C}_{15} \mathrm{H}_{9} \mathrm{ClONa}[\mathrm{M}+\mathrm{Na}]^{+} 263.0240$, found 263.0243 . 
2-(p-Tolylethynyl)benzaldehyde (1D). ${ }^{1} \mathrm{H}-\mathrm{NMR}\left(400 \mathrm{MHz}, \mathrm{CDCl}_{3}\right) \delta 10.68(\mathrm{~s}, 1 \mathrm{H}), 7.97(\mathrm{~d}, J=7.2 \mathrm{~Hz}$, $1 \mathrm{H}), 7.66(\mathrm{~d}, J=7.2 \mathrm{~Hz}, 1 \mathrm{H}), 7.62(\mathrm{~m}, 1 \mathrm{H}), 7.49(\mathrm{~m}, 3 \mathrm{H}), 7.23(\mathrm{~d}, J=8.0 \mathrm{~Hz}, 2 \mathrm{H}), 2.42(\mathrm{~s}, 3 \mathrm{H})$. ${ }^{13} \mathrm{C}-\mathrm{NMR}\left(125 \mathrm{MHz}, \mathrm{CDCl}_{3}\right) \delta 191.87,139.42,135.77,133.81,133.17,131.62,129.34,128.45$, 127.21, 119.27, 96.69, 84.35, 21.63. LRMS (ESI) m/z $221[\mathrm{M}+\mathrm{H}]^{+}$; HRMS (ESI) $\mathrm{m} / \mathrm{z}$ calcd $\mathrm{C}_{16} \mathrm{H}_{12} \mathrm{ONa}[\mathrm{M}+\mathrm{Na}]^{+} 243.0786$, found 243.0784.

2-(m-Tolylethynyl)benzaldehyde (1E). ${ }^{1} \mathrm{H}-\mathrm{NMR}\left(500 \mathrm{MHz}, \mathrm{CDCl}_{3}\right) \delta 10.66(\mathrm{~s}, 1 \mathrm{H}), 7.95(\mathrm{~d}, J=7.8 \mathrm{~Hz}$, $1 \mathrm{H}), 7.91(\mathrm{~d}, J=9.7 \mathrm{~Hz}, 1 \mathrm{H}), 7.64(\mathrm{~d}, J=7.5 \mathrm{~Hz}, 1 \mathrm{H}), 7.59(\mathrm{td}, J=7.6,1.1 \mathrm{~Hz}, 1 \mathrm{H}), 7.45(\mathrm{t}, J=7.6 \mathrm{~Hz}$, 1H), 7.44-7.35 (m, 3H), 7.29 (d, $J=7.6 \mathrm{~Hz}, 1 \mathrm{H}), 7.21(\mathrm{~d}, J=7.7 \mathrm{~Hz}, 1 \mathrm{H}), 2.38(\mathrm{~s}, 3 \mathrm{H}) .{ }^{13} \mathrm{C}-\mathrm{NMR}$ $\left(125 \mathrm{MHz} \mathrm{CDCl}_{3}\right) \delta 191.78,138.19,135.70,133.73,133.11,132.16,129.92,128.67,128.45,128.34$, 127.15, 126.96, 122.02, 96.52, 84.44, 21.15. LRMS (ESI) $\mathrm{m} / z 221[\mathrm{M}+\mathrm{H}]^{+}$; HRMS (ESI) $\mathrm{m} / z$ calcd $\mathrm{C}_{16} \mathrm{H}_{12} \mathrm{ONa}[\mathrm{M}+\mathrm{Na}]^{+}$243.0786, found 243.0791.

5-Fluoro-2-(phenylethynyl)benzaldehyde (1F). ${ }^{1} \mathrm{H}-\mathrm{NMR}\left(400 \mathrm{MHz}, \mathrm{CDCl}_{3}\right) \delta 10.63(\mathrm{~s}, 1 \mathrm{H}), 7.71-7.62$ $(\mathrm{m}, 2 \mathrm{H}), 7.61-7.53(\mathrm{~m}, 2 \mathrm{H}), 7.43-7.41(\mathrm{~m}, 3 \mathrm{H}), 7.38-7.28(\mathrm{~m}, 1 \mathrm{H}) .{ }^{13} \mathrm{C}-\mathrm{NMR}\left(125 \mathrm{MHz}, \mathrm{CDCl}_{3}\right) \delta$ $190.52,162.41(\mathrm{~d}, J=252.7 \mathrm{~Hz}), 137.78(\mathrm{~d}, J=6.6 \mathrm{~Hz}), 135.28(\mathrm{~d}, J=7.6 \mathrm{~Hz}), 131.67,129.21$, 128.60, 123.04 (d, $J=3.3 \mathrm{~Hz}), 122.13,121.42$ (d, $J=22.8 \mathrm{~Hz}), 113.73$ (d, $J=23.0 \mathrm{~Hz}), 96.06,83.84$. LRMS (ESI) $m / z 225[\mathrm{M}+\mathrm{H}]^{+}$; HRMS (ESI) $\mathrm{m} / z$ calcd $\mathrm{C}_{15} \mathrm{H}_{9} \mathrm{ONaF}[\mathrm{M}+\mathrm{Na}]^{+}$247.0535, found 247.0529 .

4-Methyl-2-(phenylethynyl)benzaldehyde (1G). ${ }^{1} \mathrm{H}-\mathrm{NMR}\left(400 \mathrm{MHz}, \mathrm{CDCl}_{3}\right) \delta 10.62(\mathrm{~s}, 1 \mathrm{H}), 7.88(\mathrm{~d}$, $J=8.0 \mathrm{~Hz}, 1 \mathrm{H}), 7.64-7.55(\mathrm{~m}, 2 \mathrm{H}), 7.49(\mathrm{~s}, 1 \mathrm{H}), 7.46-7.36(\mathrm{~m}, 3 \mathrm{H}), 7.30(\mathrm{~s}, 1 \mathrm{H}), 2.45(\mathrm{~s}, 3 \mathrm{H})$. ${ }^{13} \mathrm{C}-\mathrm{NMR}\left(125 \mathrm{MHz}, \mathrm{CDCl}_{3}\right) \delta 191.43,144.91,133.69,133.62,131.70,129.72,129.03,128.55$, 127.36, 126.91, 122.45, 95.87, 85.13, 21.67. LRMS (ESI) $\mathrm{m} / z 221[\mathrm{M}+\mathrm{H}]^{+}$; HRMS (ESI) $\mathrm{m} / z$ calcd $\mathrm{C}_{16} \mathrm{H}_{12} \mathrm{ONa}[\mathrm{M}+\mathrm{Na}]^{+}$243.0786, found 243.0786.

2-(Oct-1-yn-1-yl)benzaldehyde (1H). ${ }^{1} \mathrm{H}-\mathrm{NMR}\left(400 \mathrm{MHz}, \mathrm{CDCl}_{3}\right) \delta 10.56(\mathrm{~s}, 1 \mathrm{H}), 7.91(\mathrm{~d}, J=8.0 \mathrm{~Hz}$, $1 \mathrm{H}), 7.65-7.47(\mathrm{~m}, 2 \mathrm{H}), 7.47-7.33(\mathrm{~m}, 1 \mathrm{H}), 2.50(\mathrm{t}, J=7.1 \mathrm{~Hz}, 2 \mathrm{H}), 1.73-1.59(\mathrm{~m}, 2 \mathrm{H}), 1.54-1.44(\mathrm{~m}$, 2H), $1.42-1.31(\mathrm{~m}, 4 \mathrm{H}), 0.93(\mathrm{t}, J=6.8 \mathrm{~Hz}, 3 \mathrm{H}) .{ }^{13} \mathrm{C}-\mathrm{NMR}\left(125 \mathrm{MHz}, \mathrm{CDCl}_{3}\right) \delta 192.21,135.96$, 133.70, 133.29, 127.99, 127.84, 126.88, 98.24, 76.32, 31.32, 28.67, 28.50, 22.56, 19.61, 14.07. LRMS (ESI) $m / z 215[\mathrm{M}+\mathrm{H}]^{+}$; HRMS (ESI) $m / z$ calcd $\mathrm{C}_{15} \mathrm{H}_{18} \mathrm{ONa}[\mathrm{M}+\mathrm{Na}]^{+} 237.1255$, found 237.1259.

\subsection{General Procedure for Synthesis of Dihydrobenzo [4,5][1,3]oxazino[2,3-a]isoquinolines}

\section{(3Aa as an Example)}

To a solution of 2-(phenylethynyl)benzaldehyde $(\mathbf{1 A}, 0.1 \mathrm{mmol})$ in dry toluene $(2 \mathrm{~mL})$ were added (2-aminophenyl)methanol (2a, $0.12 \mathrm{mmol})$ and $\mathrm{AgNO}_{3}$ catalyst $(5 \mathrm{~mol} \%)$. Then, the reaction vial was sealed and the mixture was heated to $80{ }^{\circ} \mathrm{C}$ for $3 \mathrm{~h}$. Afterwards, the cooled mixture was concentrated under reduced pressure, and the resulting reside was purified by flash column chromatography $\left(\mathrm{PE} / \mathrm{EA}=30 / 1, \mathrm{v} / \mathrm{v}\right.$, as an eluent) to afford the desired product 3Aa [17] in 94\% yield. ${ }^{1} \mathrm{H}-\mathrm{NMR}$ $\left(400 \mathrm{MHz}, \mathrm{CDCl}_{3}\right) \delta 7.43(\mathrm{~d}, J=7.6 \mathrm{~Hz}, 1 \mathrm{H}), 7.34(\mathrm{t}, J=7.2 \mathrm{~Hz}, 1 \mathrm{H}), 7.25-7.19(\mathrm{~m}, 7 \mathrm{H}), 7.06$ $(\mathrm{d}, J=7.6 \mathrm{~Hz}, 1 \mathrm{H}), 6.93(\mathrm{t}, J=7.6 \mathrm{~Hz}, 1 \mathrm{H}), 6.80(\mathrm{t}, J=8.4 \mathrm{~Hz}, 1 \mathrm{H}), 6.24(\mathrm{~d}, J=8.0 \mathrm{~Hz}, 1 \mathrm{H}), 6.10$ (s, 1H), 5.97 (s, 1H), 5.27 (d, $J=14.4 \mathrm{~Hz}, 1 \mathrm{H}), 5.11(\mathrm{~d}, J=14.8 \mathrm{~Hz}, 1 \mathrm{H}) .{ }^{13} \mathrm{C}-\mathrm{NMR}(100 \mathrm{MHz}$, 
$\left.\mathrm{CDCl}_{3}\right) \delta 140.75,139.82,136.90,132.32,128.96,128.56,128.41,127.95,127.82,126.75,125.97$, 125.73, 124.66, 124.29, 123.74, 122.51, 105.63, 84.96, 69.99. LRMS (EI) $m / z 311\left(\mathrm{M}^{+}\right)$; HRMS (EI) $\mathrm{m} / \mathrm{z}$ calcd $\mathrm{C}_{22} \mathrm{H}_{17} \mathrm{NO}\left(\mathrm{M}^{+}\right) 311.1310$, found 311.1303 .

8-Fluoro-12-phenyl-4b,6-dihydrobenzo[4,5][1,3]oxazino[2,3-a]isoquinoline (3Ab). ${ }^{1} \mathrm{H}-\mathrm{NMR}$ (400 MHz, $\left.\mathrm{CDCl}_{3}\right) \delta 7.40(\mathrm{~d}, J=7.2 \mathrm{~Hz}, 1 \mathrm{H}), 7.34(\mathrm{t}, J=7.6 \mathrm{~Hz}, 1 \mathrm{H}), 7.26-7.22(\mathrm{~m}, 4 \mathrm{H}), 7.20-7.17(\mathrm{~m}, 3 \mathrm{H}), 6.77$ $(\mathrm{dd}, J=8.4,2.4 \mathrm{~Hz}, 1 \mathrm{H}), 6.51(\mathrm{td}, J=8.8,2.8 \mathrm{~Hz}, 1 \mathrm{H}), 6.19(\mathrm{dd}, J=8.8,4.8 \mathrm{~Hz}, 1 \mathrm{H}), 6.04(\mathrm{~s}, 1 \mathrm{H})$, $5.91(\mathrm{~s}, 1 \mathrm{H}), 5.24(\mathrm{~d}, J=15.2 \mathrm{~Hz}, 1 \mathrm{H}), 5.07(\mathrm{~d}, J=15.2 \mathrm{~Hz}, 1 \mathrm{H}) .{ }^{13} \mathrm{C}-\mathrm{NMR}\left(100 \mathrm{MHz}, \mathrm{CDCl}_{3}\right) \delta$ $158.60(\mathrm{~d}, J=242.0 \mathrm{~Hz}), 140.87,136.70,136.01,132.17,130.13$ (d, $J=7.4 \mathrm{~Hz}), 129.17,128.74$, 127.98, 127.89, 127.15, 125.93, 125.60 (d, $J=7.8 \mathrm{~Hz}), 124.34,112.81$ (d, $J=22.8 \mathrm{~Hz}), 111.15$ (d, $J=22.9 \mathrm{~Hz}$ ), 104.87, 84.98, 67.69. LRMS (EI) m/z $329\left(\mathrm{M}^{+}\right)$; HRMS (EI) $m / z$ calcd $\mathrm{C}_{22} \mathrm{H}_{16} \mathrm{FNO}\left(\mathrm{M}^{+}\right)$ 329.1216 , found 329.1213 .

8-Chloro-12-phenyl-4b,6-dihydrobenzo[4,5][1,3]oxazino[2,3-a]isoquinoline (3Ac). ${ }^{1} \mathrm{H}-\mathrm{NMR}$ (400 MHz, $\left.\mathrm{CDCl}_{3}\right) \delta 7.41(\mathrm{~d}, J=7.6 \mathrm{~Hz}, 1 \mathrm{H}), 7.33(\mathrm{td}, J=7.6,1.2 \mathrm{~Hz}, 1 \mathrm{H}), 7.29-7.17(\mathrm{~m}, 6 \mathrm{H}), 7.04(\mathrm{~d}, J=2.4 \mathrm{~Hz}$, $1 \mathrm{H}), 6.75(\mathrm{dd}, J=8.8,2.4 \mathrm{~Hz}, 1 \mathrm{H}), 6.13(\mathrm{~d}, J=8.8 \mathrm{~Hz}, 1 \mathrm{H}), 6.05(\mathrm{~s}, 1 \mathrm{H}), 6.0(\mathrm{~s}, 1 \mathrm{H}), 5.19(\mathrm{~d}, J=14.4 \mathrm{~Hz}$, $1 \mathrm{H}), 5.04(\mathrm{~d}, J=14.4 \mathrm{~Hz}, 1 \mathrm{H}) .{ }^{13} \mathrm{C}-\mathrm{NMR}\left(100 \mathrm{MHz}, \mathrm{CDCl}_{3}\right) \delta 140.19,138.24,136.43,132.10$, $129.55,129.05,128.46,128.16,128.05,127.54,126.80,126.50,126.24,126.00,124.61,124.51$, 124.40, 106.49, 85.05, 67.44. LRMS (EI) $m / z 345\left(\mathrm{M}^{+}\right)$; HRMS (EI) $m / z$ calcd $\mathrm{C}_{22} \mathrm{H}_{16} \mathrm{ClNO}\left(\mathrm{M}^{+}\right.$) 345.0920 , found 345.0919 .

9-Chloro-12-phenyl-4b,6-dihydrobenzo[4,5][1,3] oxazino[2,3-a] isoquinoline (3Ad). ${ }^{1} \mathrm{H}-\mathrm{NMR}$ (300 MHz, $\left.\mathrm{CDCl}_{3}\right) \delta 7.43(\mathrm{~d}, J=7.2 \mathrm{~Hz}, 1 \mathrm{H}), 7.36-7.26(\mathrm{~m}, 6 \mathrm{H}), 7.23-7.18(\mathrm{~m}, 2 \mathrm{H}), 6.97(\mathrm{~d}, J=8.1 \mathrm{~Hz}, 1 \mathrm{H})$, $6.84(\mathrm{dd}, J=8.1,1.8 \mathrm{~Hz}, 1 \mathrm{H}), 6.16(\mathrm{~d}, J=1.5 \mathrm{~Hz}, 1 \mathrm{H}), 6.11(\mathrm{~s}, 1 \mathrm{H}), 6.07(\mathrm{~s}, 1 \mathrm{H}), 5.16(\mathrm{~d}, J=14.1 \mathrm{~Hz}, 1 \mathrm{H})$, $5.04(\mathrm{~d}, J=14.1 \mathrm{~Hz}, 1 \mathrm{H}) .{ }^{13} \mathrm{C}-\mathrm{NMR}\left(100 \mathrm{MHz}, \mathrm{CDCl}_{3}\right) \delta 139.88,139.37,135.60,131.61,131.20$, $128.49,127.85,127.78,127.29,125.99,125.70,125.50,125.06,123.94,122.11,121.49,107.48$, 84.64, 66.85. LRMS (EI) $m / z 345\left(\mathrm{M}^{+}\right)$; HRMS (EI) $m / z$ calcd $\mathrm{C}_{22} \mathrm{H}_{16} \mathrm{ClNO}\left(\mathrm{M}^{+}\right)$345.0920, found 345.0916 .

8-Bromo-12-phenyl-4b,6-dihydrobenzo[4,5][1,3] oxazino[2,3-a]isoquinoline (3Ae). ${ }^{1} \mathrm{H}-\mathrm{NMR}$ (400 MHz, $\left.\mathrm{CDCl}_{3}\right) \delta 7.41(\mathrm{~d}, J=8.0 \mathrm{~Hz}, 1 \mathrm{H}), 7.32(\mathrm{td}, J=7.6,1.2 \mathrm{~Hz}, 1 \mathrm{H}), 7.21-7.28(\mathrm{~m}, 6 \mathrm{H}), 7.19-7.17(\mathrm{~m}$, 2H), $6.88(\mathrm{dd}, J=8.4,2.0 \mathrm{~Hz}, 1 \mathrm{H}), 6.07(\mathrm{~s}, 1 \mathrm{H}), 6.05(\mathrm{~s}, 1 \mathrm{H}), 6.01(\mathrm{~s}, 1 \mathrm{H}), 5.18(\mathrm{~d}, J=14.8 \mathrm{~Hz}, 1 \mathrm{H})$, $5.03(\mathrm{~d}, J=14.8 \mathrm{~Hz}, 1 \mathrm{H}) .{ }^{13} \mathrm{C}-\mathrm{NMR}\left(100 \mathrm{MHz}, \mathrm{CDCl}_{3}\right) \delta 140.07,138.69,136.38,132.11,129.89$, $129.04,128.93,128.42,128.21,128.09,127.55,126.98,126.39,126.32,124.71,124.43,115.04$, 106.83, 85.07, 67.33. LRMS (EI) $m / z 389\left(\mathrm{M}^{+}\right)$; HRMS (EI) $m / z$ calcd $\mathrm{C}_{22} \mathrm{H}_{16} \mathrm{BrNO}\left(\mathrm{M}^{+}\right)$389.0415, found 389.0410 .

8-Fluoro-12-(4-fluorophenyl)-4b,6-dihydrobenzo[4,5][1,3]oxazino[2,3-a]isoquinoline (3Bb). ${ }^{1} \mathrm{H}-\mathrm{NMR}$ $\left(400 \mathrm{MHz}, \mathrm{CDCl}_{3}\right) \delta 7.43(\mathrm{~d}, J=7.6 \mathrm{~Hz}, 1 \mathrm{H}), 7.37(\mathrm{t}, J=7.2 \mathrm{~Hz}, 1 \mathrm{H}), 7.25-7.30(\mathrm{~m}, 1 \mathrm{H}), 7.15-7.23$ (m, 3H), 6.97 (t, $J=8.4 \mathrm{~Hz}, 2 \mathrm{H}), 6.8(\mathrm{dd}, J=8.4,2.4 \mathrm{~Hz}, 1 \mathrm{H}), 6.57$ (td, $J=8.8,2.4 \mathrm{~Hz}, 1 \mathrm{H}), 6.21$ (dd, $J=8.8,4.8 \mathrm{~Hz}, 1 \mathrm{H}), 6.05$ (s, 1H), $5.90(\mathrm{~s}, 1 \mathrm{H}), 5.27$ (d, $J=15.2 \mathrm{~Hz}, 1 \mathrm{H}), 5.09$ (d, $J=15.2 \mathrm{~Hz}, 1 \mathrm{H})$. ${ }^{13} \mathrm{C}-\mathrm{NMR}\left(100 \mathrm{MHz}, \mathrm{CDCl}_{3}\right) \delta 162.39(\mathrm{~d}, J=246.6 \mathrm{~Hz}), 158.75(\mathrm{~d}, J=242.7 \mathrm{~Hz}), 139.96,135.91$, 132.84, 132.09, 130.57 (d, $J=8.0 \mathrm{~Hz}), 130.36$ (d, $J=7.1 \mathrm{~Hz}), 129.27,127.22,126.11,126.04,125.71$ 
$(\mathrm{d}, J=8.1 \mathrm{~Hz}), 124.40,115.09(\mathrm{~d}, J=21.4 \mathrm{~Hz}), 113.00(\mathrm{~d}, J=23.0 \mathrm{~Hz}), 111.34(\mathrm{~d}, J=22.8 \mathrm{~Hz})$, 105.09, 85.07, 67.74. LRMS (EI) $m / z 347\left(\mathrm{M}^{+}\right.$); HRMS (EI) $m / z$ calcd $\mathrm{C}_{22} \mathrm{H}_{15} \mathrm{~F}_{2} \mathrm{NO}\left(\mathrm{M}^{+}\right.$) 347.1122, found 347.1116 .

9-Chloro-12-(4-fluorophenyl)-4b,6-dihydrobenzo[4,5][1,3]oxazino[2,3-a]isoquinoline (3Bd). ${ }^{1} \mathrm{H}-\mathrm{NMR}$ $\left(400 \mathrm{MHz}, \mathrm{CDCl}_{3}\right) \delta 7.45(\mathrm{~d}, J=7.6 \mathrm{~Hz}, 1 \mathrm{H}), 7.36(\mathrm{td}, J=7.6,1.2 \mathrm{~Hz}, 1 \mathrm{H}), 731-7.25(\mathrm{~m}, 3 \mathrm{H}), 7.22(\mathrm{~d}$, $J=7.6 \mathrm{~Hz}, 1 \mathrm{H}), 7.05-6.98(\mathrm{~m}, 3 \mathrm{H}), 6.89(\mathrm{dd}, J=8.0,2.0 \mathrm{~Hz}, 1 \mathrm{H}), 6.18(\mathrm{~d}, J=1.6 \mathrm{~Hz}, 1 \mathrm{H}), 6.10$ (s, $1 \mathrm{H}), 6.07(\mathrm{~s}, 1 \mathrm{H}), 5.16(\mathrm{~d}, J=14.4 \mathrm{~Hz}, 1 \mathrm{H}), 5.05(\mathrm{~d}, J=14.4 \mathrm{~Hz}, 1 \mathrm{H}) .{ }^{13} \mathrm{C}-\mathrm{NMR}\left(100 \mathrm{MHz}, \mathrm{CDCl}_{3}\right) \delta$ $162.63(\mathrm{~d}, J=246.8 \mathrm{~Hz}), 140.26,138.91,132.24(\mathrm{~d}, J=3.4 \mathrm{~Hz}), 132.00,131.88,130.05(\mathrm{~d}, J=8.2 \mathrm{~Hz})$, $129.02,127.76,126.66,126.26,126.01,125.75,124.52,122.67,122.10,115.40$ (d, $J=21.6 \mathrm{~Hz})$, 108.06, 85.08, 67.37. LRMS (EI) $m / z 363\left(\mathrm{M}^{+}\right)$; HRMS (EI) $m / z$ calcd $\mathrm{C}_{22} \mathrm{H}_{15} \mathrm{ClFNO}\left(\mathrm{M}^{+}\right.$) 363.0826, found 363.0831 .

8-Bromo-12-(4-fluorophenyl)-4b,6-dihydrobenzo[4,5][1,3]oxazino[2,3-a]isoquinoline (3Be). ${ }^{1} \mathrm{H}-\mathrm{NMR}$ $\left(400 \mathrm{MHz}, \mathrm{CDCl}_{3}\right) \delta 7.45(\mathrm{~d}, J=7.6 \mathrm{~Hz}, 1 \mathrm{H}), 7.36(\mathrm{td}, J=7.6,1.2 \mathrm{~Hz}, 1 \mathrm{H}), 7.31-7.25(\mathrm{~m}, 3 \mathrm{H}), 7.22$ $(\mathrm{d}, J=7.6 \mathrm{~Hz}, 1 \mathrm{H}), 7.05-6.98(\mathrm{~m}, 3 \mathrm{H}), 6.89(\mathrm{dd}, J=8.0,2.0 \mathrm{~Hz}, 1 \mathrm{H}), 6.18(\mathrm{~d}, J=1.6 \mathrm{~Hz}, 1 \mathrm{H}), 6.10$ (s, $1 \mathrm{H}), 6.07(\mathrm{~s}, 1 \mathrm{H}), 5.16(\mathrm{~d}, J=14.4 \mathrm{~Hz}, 1 \mathrm{H}), 5.05(\mathrm{~d}, J=14.4 \mathrm{~Hz}, 1 \mathrm{H}) .{ }^{13} \mathrm{C}-\mathrm{NMR}\left(100 \mathrm{MHz}, \mathrm{CDCl}_{3}\right) \delta$ $162.49(\mathrm{~d}, J=246.8 \mathrm{~Hz}), 139.13,138.57,132.51(\mathrm{~d}, J=3.2 \mathrm{~Hz}), 132.01,130.27,130.19,130.1$, $129.09(\mathrm{~d}, J=6.4 \mathrm{~Hz}), 127.72,126.99,126.49,126.46,124.80,124.47,115.42115 .26$ (d, $J=21.6 \mathrm{~Hz})$, 106.91, 85.11, 67.35. LRMS (EI) $m / z 407\left(\mathrm{M}^{+}\right)$; HRMS (EI) $m / z$ calcd $\mathrm{C}_{22} \mathrm{H}_{15} \mathrm{BrFNO}\left(\mathrm{M}^{+}\right)$407.0321, found 407.0320 .

12-(4-Fluorophenyl)-8-methyl-4b,6-dihydrobenzo[4,5][1,3]oxazino[2,3-a]isoquinoline (3Bf). ${ }^{1} \mathrm{H}-\mathrm{NMR}$ $\left(400 \mathrm{MHz}, \mathrm{CDCl}_{3}\right) \delta 7.40(\mathrm{~d}, J=7.6 \mathrm{~Hz}, 1 \mathrm{H}), 7.33(\mathrm{t}, J=7.2 \mathrm{~Hz}, 1 \mathrm{H}), 7.26-7.14(\mathrm{~m}, 4 \mathrm{H}), 6.93$ (t, $J=8.8 \mathrm{~Hz}, 2 \mathrm{H}), 6.86(\mathrm{~s}, 1 \mathrm{H}), 6.63(\mathrm{~d}, J=8.0 \mathrm{~Hz}, 1 \mathrm{H}), 6.12(\mathrm{~d}, J=8.4 \mathrm{~Hz}, 1 \mathrm{H}), 6.05(\mathrm{~s}, 1 \mathrm{H}), 5.84(\mathrm{~s}$, $1 \mathrm{H}), 5.25(\mathrm{~d}, J=14.8 \mathrm{~Hz}, 1 \mathrm{H}), 5.06(\mathrm{~d}, J=14.8 \mathrm{~Hz}, 1 \mathrm{H}), 2.25(\mathrm{~s}, 3 \mathrm{H}) .{ }^{13} \mathrm{C}-\mathrm{NMR}\left(100 \mathrm{MHz}, \mathrm{CDCl}_{3}\right) \delta$ $162.26(\mathrm{~d}, J=246.1 \mathrm{~Hz}), 140.12,137.29,133.14(\mathrm{~d}, J=3.7 \mathrm{~Hz}), 132.69,132.20,130.48(\mathrm{~d}, J=8.3 \mathrm{~Hz})$, $129.06,128.55,127.19,126.48,126.10,125.84,125.16,124.21,124.07,114.88(\mathrm{~d}, J=21.4 \mathrm{~Hz})$, 104.47, 84.93, 68.05, 20.81. LRMS (EI) $m / z 343\left(\mathrm{M}^{+}\right)$; HRMS (EI) $m / z$ calcd $\mathrm{C}_{23} \mathrm{H}_{18} \mathrm{FNO}\left(\mathrm{M}^{+}\right.$) 343.1372 , found 343.1375 .

12-(4-Fluorophenyl)-10-methyl-4b,6-dihydrobenzo[4,5][1,3]oxazino[2,3-a]isoquinoline (3Bg). ${ }^{1} \mathrm{H}-\mathrm{NMR}$ $\left(400 \mathrm{MHz}, \mathrm{CDCl}_{3}\right) \delta 7.38-7.36(\mathrm{~m}, 2 \mathrm{H}), 7.26-7.20(\mathrm{~m}, 2 \mathrm{H}), 7.10-6.92(\mathrm{~m}, 4 \mathrm{H}), 6.87-6.77(\mathrm{~m}, 3 \mathrm{H})$, 5.91-5.90 (m, 2H), 5.30-5.17 (m, 2H), $1.67(\mathrm{~s}, 3 \mathrm{H}) .{ }^{13} \mathrm{C}-\mathrm{NMR}\left(125 \mathrm{MHz}, \mathrm{CDCl}_{3}\right) \delta 162.21(\mathrm{~d}$, $J=246.3 \mathrm{~Hz}), 141.92,140.45,133.85,133.35,132.50,130.99,130.16$ (d, $J=8.0 \mathrm{~Hz}), 129.36,128.50$, 127.80, $125.66(\mathrm{~d}, J=33.1 \mathrm{~Hz}), 125.67,124.41,122.70,114.53,114.36,104.47,85.02,67.75,17.34$. LRMS (EI) $m / z 343\left(\mathrm{M}^{+}\right)$; HRMS (EI) $m / z$ calcd $\mathrm{C}_{23} \mathrm{H}_{18} \mathrm{FNO}\left(\mathrm{M}^{+}\right)$343.1372, found 343.1368.

12-(4-Chlorophenyl)-4b,6-dihydrobenzo[4,5][1,3] oxazino[2,3-a]isoquinoline (3Ca). ${ }^{1} \mathrm{H}-\mathrm{NMR}$ (400 MHz, $\left.\mathrm{CDCl}_{3}\right) \delta 7.42(\mathrm{~d}, J=7.6 \mathrm{~Hz}, 1 \mathrm{H}), 7.33(\mathrm{t}, J=7.2 \mathrm{~Hz}, 1 \mathrm{H}), 7.27-7.14(\mathrm{~m}, 6 \mathrm{H}), 7.06(\mathrm{~d}, J=7.2 \mathrm{~Hz}$, $1 \mathrm{H}), 6.95(\mathrm{t}, J=7.6 \mathrm{~Hz}, 1 \mathrm{H}), 6.84(\mathrm{t}, J=7.6 \mathrm{~Hz}, 1 \mathrm{H}), 6.22(\mathrm{~d}, J=8.4 \mathrm{~Hz}, 1 \mathrm{H}), 6.08(\mathrm{~s}, 1 \mathrm{H}), 5.95$ (s, $1 \mathrm{H}), 5.26(\mathrm{~d}, J=14.4 \mathrm{~Hz}, 1 \mathrm{H}), 5.09(\mathrm{~d}, J=14.8 \mathrm{~Hz}, 1 \mathrm{H}) .{ }^{13} \mathrm{C}-\mathrm{NMR}\left(100 \mathrm{MHz}, \mathrm{CDCl}_{3}\right) \delta 139.52$, $135.38,133.71,132.04,129.81,129.02,128.44,128.22,126.76,126.23,125.95,124.81,124.37$, 
123.67, 122.73, 106.07, 84.91, 67.97. LRMS (EI) $m / z 345\left(\mathrm{M}^{+}\right.$); HRMS (EI) $\mathrm{m} / z$ calcd $\mathrm{C}_{22} \mathrm{H}_{16} \mathrm{ClNO}$ $\left(\mathrm{M}^{+}\right)$345.0920, found 345.0890.

8-Chloro-12-(4-chlorophenyl)-4b,6-dihydrobenzo[4,5][1,3]oxazino[2,3-a]isoquinoline (3Cc). ${ }^{1} \mathrm{H}-\mathrm{NMR}$ $\left(400 \mathrm{MHz}, \mathrm{CDCl}_{3}\right) \delta 7.41(\mathrm{~d}, J=7.6 \mathrm{~Hz}, 1 \mathrm{H}), 7.34$ (td, $\left.J=7.2,1.2 \mathrm{~Hz}, 1 \mathrm{H}\right), 7.28-7.23(\mathrm{~m}, 3 \mathrm{H})$, $7.20-7.14(\mathrm{~m}, 3 \mathrm{H}), 7.06(\mathrm{~d}, J=2.0 \mathrm{~Hz}, 1 \mathrm{H}), 6.80(\mathrm{dd}, J=8.8,2.4 \mathrm{~Hz}, 1 \mathrm{H}), 6.12(\mathrm{~d}, J=8.4 \mathrm{~Hz}, 1 \mathrm{H})$, $6.04(\mathrm{~s}, 1 \mathrm{H}), 5.99(\mathrm{~s}, 1 \mathrm{H}), 5.18(\mathrm{~d}, J=14.8 \mathrm{~Hz}, 1 \mathrm{H}), 5.04(\mathrm{~d}, J=14.8 \mathrm{~Hz}, 1 \mathrm{H}) .{ }^{13} \mathrm{C}-\mathrm{NMR}(100 \mathrm{MHz}$, CDCl3) $\delta 138.99,137.96,134.93,133.99,131.85,129.71,129.62,129.12,128.45,127.80,126.92$, 126.54, 126.51, 126.24, 124.79, 124.51, 124.42, 107.02, 85.05, 67.45. LRMS (EI) $\mathrm{m} / z 379\left(\mathrm{M}^{+}\right)$; HRMS (EI) $m / z$ calcd $\mathrm{C}_{22} \mathrm{H}_{15} \mathrm{Cl}_{2} \mathrm{NO}\left(\mathrm{M}^{+}\right) 379.0531$, found 379.0503 .

9-Chloro-12-(4-chlorophenyl)-4b,6-dihydrobenzo[4,5][1,3]oxazino[2,3-a] isoquinoline (3Cd). ${ }^{1} \mathrm{H}-\mathrm{NMR}$ $\left(400 \mathrm{MHz}, \mathrm{CDCl}_{3}\right) \delta 7.42(\mathrm{~d}, J=7.6 \mathrm{~Hz}, 1 \mathrm{H}), 7.33(\mathrm{td}, J=7.2,1.2 \mathrm{~Hz}, 1 \mathrm{H}), 7.29-7.25(\mathrm{~m}, 3 \mathrm{H})$, $7.23-7.17(\mathrm{~m}, 3 \mathrm{H}), 6.97(\mathrm{~d}, J=8.0 \mathrm{~Hz}, 1 \mathrm{H}), 6.86(\mathrm{dd}, J=8.0,2.0 \mathrm{~Hz}, 1 \mathrm{H}), 6.15(\mathrm{~d}, J=1.6 \mathrm{~Hz}, 1 \mathrm{H})$, $6.11(\mathrm{~s}, 1 \mathrm{H}), 6.04(\mathrm{~s}, 1 \mathrm{H}), 5.12(\mathrm{~d}, J=14.4 \mathrm{~Hz}, 1 \mathrm{H}), 5.02(\mathrm{~d}, J=14.4 \mathrm{~Hz}, 1 \mathrm{H}) .{ }^{13} \mathrm{C}-\mathrm{NMR}(100 \mathrm{MHz}$, $\left.\mathrm{CDCl}_{3}\right) \delta 140.08,138.61,134.56,134.15,131.96$ 131.82, 129.37, 128.96, 128.57, 127.94, 126.82, 126.08, 125.83, 125.74, 124.57, 122.35, 122.00, 108.72, 84.99, 67.26. LRMS (EI) $\mathrm{m} / z 379\left(\mathrm{M}^{+}\right)$; HRMS (EI) $m / z$ calcd $\mathrm{C}_{22} \mathrm{H}_{15} \mathrm{Cl}_{2} \mathrm{NO}\left(\mathrm{M}^{+}\right) 379.0531$, found 379.0508 .

12-(p-Tolyl)-4b,6-dihydrobenzo[4,5][1,3]oxazino[2,3-a]isoquinoline (3Da). ${ }^{1} \mathrm{H}-\mathrm{NMR}$ (400 MHz, $\left.\mathrm{CDCl}_{3}\right) \delta 7.41(\mathrm{~d}, J=7.6,1 \mathrm{H}), 7.31(\mathrm{td}, J=7.6,1.2 \mathrm{~Hz}, 1 \mathrm{H}), 7.23(\mathrm{td}, J=7.2,1.2 \mathrm{~Hz}, 1 \mathrm{H}), 7.17(\mathrm{~d}$, $J=7.6 \mathrm{~Hz}, 1 \mathrm{H}), 7.12(\mathrm{~d}, J=8.0,2 \mathrm{H}), 7.05(\mathrm{~m}, 3 \mathrm{H}), 6.92(\mathrm{td}, J=7.6,1.2 \mathrm{~Hz}, 1 \mathrm{H}), 6.81(\mathrm{td}, J=8.4,1.2 \mathrm{~Hz}$, $1 \mathrm{H}), 6.25(\mathrm{~d}, J=8.0,1 \mathrm{H}), 6.08(\mathrm{~s}, 1 \mathrm{H}), 5.94(\mathrm{~s}, 1 \mathrm{H}), 5.25(\mathrm{~d}, J=14.8,1 \mathrm{H}), 5.09(\mathrm{~d}, J=14.8,1 \mathrm{H}), 2.33$ (s, 3H). ${ }^{13} \mathrm{C}-\mathrm{NMR}(100 \mathrm{MHz}, \mathrm{CDCl} 3) \delta 140.77,139.87,137.69,133.96,132.44,128.91,128.66$, $128.41,128.35,126.71,126.63,125.85,125.77,124.62,124.20,123.74,122.37,105.42,84.98,67.96$, 21.26. LRMS (EI) $m / z 325\left(\mathrm{M}^{+}\right)$; HRMS (EI) $m / z$ calcd $\mathrm{C}_{23} \mathrm{H}_{19} \mathrm{NO}\left(\mathrm{M}^{+}\right) 325.1467$, found 325.1472 .

8-Chloro-12-(p-tolyl)-4b,6-dihydrobenzo[4,5][1,3] oxazino[2,3-a]isoquinoline (3Dc). ${ }^{1} \mathrm{H}-\mathrm{NMR}$ (300 MHz, $\left.\mathrm{CDCl}_{3}\right) \delta 7.41(\mathrm{~d}, J=10.0 \mathrm{~Hz}, 1 \mathrm{H}), 7.32(\mathrm{td}, J=10.0,1.6 \mathrm{~Hz}, 1 \mathrm{H}), 7.26-7.04(\mathrm{~m}, 7 \mathrm{H}), 6.77(\mathrm{dd}$, $J=10.6,3.2 \mathrm{~Hz}, 1 \mathrm{H}), 6.16(\mathrm{~d}, J=10.6 \mathrm{~Hz}, 1 \mathrm{H}), 6.05(\mathrm{~s}, 1 \mathrm{H}), 5.99(\mathrm{~s}, 1 \mathrm{H}), 5.18(\mathrm{~d}, J=19.6 \mathrm{~Hz}, 1 \mathrm{H})$, $5.04(\mathrm{~d}, J=19.2 \mathrm{~Hz}, 1 \mathrm{H}), 2.35(\mathrm{~s}, 3 \mathrm{H}) .{ }^{13} \mathrm{C}-\mathrm{NMR}(100 \mathrm{MHz}, \mathrm{CDCl} 3) \delta 140.27,138.36,138.04$, $133.56,132.31,129.53,129.03,128.92,128.36,127.42,127.00,126.41,126.17,126.11,124.61$, 124.53, 124.37, 106.37, 85.17, 67.45, 21.30. LRMS (EI) $m / z 359\left(\mathrm{M}^{+}\right)$; HRMS (EI) $\mathrm{m} / z$ calcd $\mathrm{C}_{23} \mathrm{H}_{18} \mathrm{ClNO}\left(\mathrm{M}^{+}\right) 359.1077$, found 359.1078.

9-Chloro-12-(p-tolyl)-4b,6-dihydrobenzo[4,5][1,3]oxazino[2,3-a]isoquinoline (3Dd). ${ }^{1} \mathrm{H}-\mathrm{NMR}$ (400 MHz, $\left.\mathrm{CDCl}_{3}\right) \delta 7.42(\mathrm{dd}, J=8.8,0.8 \mathrm{~Hz}, 1 \mathrm{H}), 7.32(\mathrm{td}, J=7.6,1.2 \mathrm{~Hz}, 1 \mathrm{H}), 7.25(\mathrm{td}, J=7.2,1.2 \mathrm{~Hz}, 1 \mathrm{H})$, $7.20-7.16(\mathrm{~m}, 3 \mathrm{H}), 7.10(\mathrm{~d}, J=8.0 \mathrm{~Hz}, 2 \mathrm{H}), 6.96(\mathrm{~d}, J=8.0 \mathrm{~Hz}, 1 \mathrm{H}), 6.83(\mathrm{dd}, J=8.0,1.6 \mathrm{~Hz}, 1 \mathrm{H})$, $6.18(\mathrm{~d}, J=2.0 \mathrm{~Hz}, 1 \mathrm{H}), 6.11(\mathrm{~s}, 1 \mathrm{H}), 6.05(\mathrm{~s}, 1 \mathrm{H}), 5.13(\mathrm{~d}, J=14.0 \mathrm{~Hz}, 1 \mathrm{H}), 5.02(\mathrm{~d}, J=14.0 \mathrm{~Hz}$, $1 \mathrm{H}), 2.35(\mathrm{~s}, 3 \mathrm{H}) .{ }^{13} \mathrm{C}-\mathrm{NMR}\left(100 \mathrm{MHz}, \mathrm{CDCl}_{3}\right) \delta 140.42,139.90,138.22,133.13,132.25,131.75$, $129.00,128.85,128.03,127.90,126.38,125.98,125.74,125.52,124.38,122.49,121.64,107.79$, 85.04, 67.25, 21.27. LRMS (EI) $\mathrm{m} / z 359\left(\mathrm{M}^{+}\right)$; HRMS (EI) $\mathrm{m} / z$ calcd $\mathrm{C}_{23} \mathrm{H}_{18} \mathrm{ClNO}\left(\mathrm{M}^{+}\right)$359.1077, found 359.1053 . 
8-Bromo-12-(p-tolyl)-4b,6-dihydrobenzo[4,5][1,3]oxazino[2,3-a]isoquinoline (3De). ${ }^{1} \mathrm{H}-\mathrm{NMR}$ (400 MHz, $\left.\mathrm{CDCl}_{3}\right) \delta 7.42(\mathrm{~d}, J=7.6 \mathrm{~Hz}, 1 \mathrm{H}), 7.32(\mathrm{td}, J=7.6,1.2 \mathrm{~Hz}, 1 \mathrm{H}), 7.24(\mathrm{td}, J=7.2,1.2 \mathrm{~Hz}, 1 \mathrm{H}), 7.86(\mathrm{~d}$, $J=8.0 \mathrm{~Hz}, 2 \mathrm{H}), 7.14(\mathrm{~d}, J=8.0 \mathrm{~Hz}, 2 \mathrm{H}), 7.08(\mathrm{~d}, J=8.0 \mathrm{~Hz}, 2 \mathrm{H}), 6.91(\mathrm{dd}, J=8.4,2.0 \mathrm{~Hz}, 1 \mathrm{H}), 6.10$ $(\mathrm{d}, J=8.8 \mathrm{~Hz}, 1 \mathrm{H}), 6.04(\mathrm{~s}, 1 \mathrm{H}), 6.02(\mathrm{~s}, 1 \mathrm{H}), 5.17(\mathrm{~d}, J=14.4 \mathrm{~Hz}, 1 \mathrm{H}), 5.03(\mathrm{~d}, J=14.8 \mathrm{~Hz}, 1 \mathrm{H})$, $2.35(\mathrm{~s}, 3 \mathrm{H}) .{ }^{13} \mathrm{C}-\mathrm{NMR}\left(100 \mathrm{MHz}, \mathrm{CDCl}_{3}\right) \delta 140.07,138.72,138.00,133.44,132.25,129.80,128.95$, 128.91, 128.25, 127.47, 127.10, 126.23, 126.17, 124.66, 124.32, 114.83, 106.64, 85.09, 67.25, 21.25. LRMS (EI) $m / z 403\left(\mathrm{M}^{+}\right)$; HRMS (EI) $m / z$ calcd $\mathrm{C}_{23} \mathrm{H}_{18} \mathrm{BrNO}\left(\mathrm{M}^{+}\right)$403.0572, found 403.0558 .

8-Methyl-12-(p-tolyl)-4b,6-dihydrobenzo[4,5][1,3] oxazino[2,3-a]isoquinoline (3Df). ${ }^{1} \mathrm{H}-\mathrm{NMR}$ (400 MHz, $\left.\mathrm{CDCl}_{3}\right) \delta 7.40(\mathrm{~d}, J=7.6 \mathrm{~Hz}, 1 \mathrm{H}), 7.32(\mathrm{td}, J=7.6,0.8 \mathrm{~Hz}, 1 \mathrm{H}), 7.22(\mathrm{td}, J=7.6,1.2 \mathrm{~Hz}, 1 \mathrm{H}), 7.17(\mathrm{~d}$, $J=7.6 \mathrm{~Hz}, 1 \mathrm{H}), 7.11(\mathrm{~d}, J=7.6 \mathrm{~Hz}, 2 \mathrm{H}), 7.05(\mathrm{~d}, J=8.0 \mathrm{~Hz}, 2 \mathrm{H}), 6.86(\mathrm{~s}, 1 \mathrm{H}), 6.62(\mathrm{~d}, J=7.2 \mathrm{~Hz}$, $1 \mathrm{H}), 6.17(\mathrm{~d}, J=8.0 \mathrm{~Hz}, 1 \mathrm{H}), 6.06(\mathrm{~s}, 1 \mathrm{H}), 5.87(\mathrm{~s}, 1 \mathrm{H}), 5.25(\mathrm{~d}, J=14.8 \mathrm{~Hz}, 1 \mathrm{H}), 5.07$ (d, $J=14.8 \mathrm{~Hz}$, $1 \mathrm{H}), 2.34(\mathrm{~s}, 3 \mathrm{H}), 2.25(\mathrm{~s}, 3 \mathrm{H}) .{ }^{13} \mathrm{C}-\mathrm{NMR}\left(100 \mathrm{MHz}, \mathrm{CDCl}_{3}\right) \delta 141.07,137.56,137.53,134.15$, $132.48,132.24,128.93,128.56,128.36,127.01,126.43,126.23,125.58,125.01,124.12,123.95$, 104.29, 84.98, 68.01, 21.25, 20.83. LRMS (EI) $m / z 339\left(\mathrm{M}^{+}\right)$; HRMS (EI) $m / z$ calcd $\mathrm{C}_{24} \mathrm{H}_{21} \mathrm{NO}\left(\mathrm{M}^{+}\right)$ 339.1623 , found 339.1609 .

14-(p-Tolyl)-4b,6-dihydronaphtho[2',3':4,5][1,3] oxazino[2,3-a]isoquinoline (3Dh). ${ }^{1} \mathrm{H}-\mathrm{NMR}$ (400 MHz, $\left.\mathrm{CDCl}_{3}\right) \delta 7.70-7.66(\mathrm{~m}, 1 \mathrm{H}), 7.54(\mathrm{~s}, 1 \mathrm{H}), 7.47(\mathrm{~d}, J=7.6 \mathrm{~Hz}, 1 \mathrm{H}), 7.34-7.30(\mathrm{~m}, 3 \mathrm{H}), 7.28-7.21(\mathrm{~m}$, $5 \mathrm{H}), 7.06(\mathrm{~d}, J=7.6 \mathrm{~Hz}, 2 \mathrm{H}), 6.53(\mathrm{~s}, 1 \mathrm{H}), 6.36(\mathrm{~s}, 1 \mathrm{H}), 6.20(\mathrm{~s}, 1 \mathrm{H}), 5.25(\mathrm{~d}, J=13.6 \mathrm{~Hz}, 1 \mathrm{H}), 5.23$ $(\mathrm{d}, J=13.6 \mathrm{~Hz}, 1 \mathrm{H}), 2.30(\mathrm{~s}, 3 \mathrm{H}) .{ }^{13} \mathrm{C}-\mathrm{NMR}\left(100 \mathrm{MHz}, \mathrm{CDCl}_{3}\right) \delta 140.13,137.98,136.77,133.25$, $132.84,132.49$, 129.39, 129.02, 128.59, 128.45, 128.07, 127.74, 127.15 , 126.80, 126.61, 125.61, 124.50, 124.20, 123.94, 123.06, 117.77, 109.94, 85.75, 67.43, 21.20. LRMS (EI) $m / z 375\left(\mathrm{M}^{+}\right)$; HRMS (EI) $\mathrm{m} / z$ calcd $\mathrm{C}_{27} \mathrm{H}_{21} \mathrm{NO}\left(\mathrm{M}^{+}\right) 375.1623$, found 375.1621 .

12-(m-Tolyl)-4b,6-dihydrobenzo[4,5][1,3]oxazino[2,3-a]isoquinoline (3Ea). ${ }^{1} \mathrm{H}-\mathrm{NMR}$ (400 MHz, $\left.\mathrm{CDCl}_{3}\right) \delta 7.43(\mathrm{~d}, J=7.2 \mathrm{~Hz}, 1 \mathrm{H}), 7.34(\mathrm{t}, J=7.2 \mathrm{~Hz}, 1 \mathrm{H}), 7.26-7.18(\mathrm{~m}, 2 \mathrm{H}), 7.14-7.04(\mathrm{~m}, 4 \mathrm{H})$, 6.99-6.91 (m, 2H), $6.82(\mathrm{t}, J=7.6 \mathrm{~Hz}, 1 \mathrm{H}), 6.26(\mathrm{~d}, J=8.0 \mathrm{~Hz}, 1 \mathrm{H}), 6.10(\mathrm{~s}, 1 \mathrm{H}), 5.97(\mathrm{~s}, 1 \mathrm{H}), 5.27$ $(\mathrm{d}, J=14.4 \mathrm{~Hz}, 1 \mathrm{H}), 5.11(\mathrm{~d}, J=14.4 \mathrm{~Hz}, 1 \mathrm{H}), 2.30(\mathrm{~s}, 3 \mathrm{H}) .{ }^{13} \mathrm{C}-\mathrm{NMR}\left(100 \mathrm{MHz}, \mathrm{CDCl}_{3}\right) \delta 140.89$, $139.82,137.60,136.77,132.36,129.12,128.90,128.54,128.28,127.69,126.70,125.85,125.72$, 125.65, 124.58, 124.21, 123.69, 122.42, 105.44, 84.93, 67.93, 21.34. LRMS (EI) $m / z 325\left(\mathrm{M}^{+}\right)$; HRMS (EI) $\mathrm{m} / z$ calcd $\mathrm{C}_{23} \mathrm{H}_{19} \mathrm{NO}\left(\mathrm{M}^{+}\right) 325.1467$, found 325.1468 .

8-Chloro-12-(m-tolyl)-4b,6-dihydrobenzo[4,5][1,3] oxazino[2,3-a]isoquinoline (3Ec). ${ }^{1} \mathrm{H}-\mathrm{NMR}$ (400 MHz, $\left.\mathrm{CDCl}_{3}\right) \delta 7.41(\mathrm{~d}, J=7.6 \mathrm{~Hz}, 1 \mathrm{H}), 7.33(\mathrm{t}, J=7.2 \mathrm{~Hz}, 1 \mathrm{H}), 7.26-7.10(\mathrm{~m}, 5 \mathrm{H}), 7.04(\mathrm{~s}, 1 \mathrm{H}), 6.96(\mathrm{~d}$, $J=6.8 \mathrm{~Hz}, 1 \mathrm{H}), 6.76(\mathrm{dd}, J=8.4,1.2 \mathrm{~Hz}, 1 \mathrm{H}), 6.15(\mathrm{~d}, J=8.8 \mathrm{~Hz}, 1 \mathrm{H}), 6.05(\mathrm{~s}, 1 \mathrm{H}), 5.99(\mathrm{~s}, 1 \mathrm{H})$, $5.19(\mathrm{~d}, J=14.4 \mathrm{~Hz}, 1 \mathrm{H}), 5.04(\mathrm{~d}, J=14.8 \mathrm{~Hz}, 1 \mathrm{H}), 2.31(\mathrm{~s}, 3 \mathrm{H}) .{ }^{13} \mathrm{C}-\mathrm{NMR}\left(100 \mathrm{MHz}, \mathrm{CDCl}_{3}\right) \delta$ $140.35,138.30,137.90,136.37,132.19,129.42,129.00,128.82,127.94,127.40,126.90,126.43$, $126.18,125.99,125.65,124.55,124.43,124.36,106.46,85.09,67.42,21.41$. LRMS (EI) $\mathrm{m} / z 359$ $\left(\mathrm{M}^{+}\right)$; HRMS (EI) $m / z$ calcd $\mathrm{C}_{23} \mathrm{H}_{18} \mathrm{ClNO}\left(\mathrm{M}^{+}\right)$359.1077, found 359.1063. 
8-Bromo-12-(m-tolyl)-4b,6-dihydrobenzo[4,5][1,3] oxazino[2,3-a]isoquinoline (3Ee). ${ }^{1} \mathrm{H}-\mathrm{NMR}$ (400 MHz, CDCl3) $\delta 7.41(\mathrm{~d}, J=7.6 \mathrm{~Hz}, 1 \mathrm{H}), 7.33(\mathrm{td}, J=7.2,1.2 \mathrm{~Hz}, 1 \mathrm{H}), 7.25(\mathrm{td}, J=7.6,1.2 \mathrm{~Hz}, 1 \mathrm{H})$, $7.20-7.08(\mathrm{~m}, 5 \mathrm{H}), 6.97(\mathrm{~d}, J=7.2 \mathrm{~Hz}, 1 \mathrm{H}), 6.90(\mathrm{dd}, J=8.8,2.0 \mathrm{~Hz}, 1 \mathrm{H}), 6.09(\mathrm{~d}, J=8.8 \mathrm{~Hz}, 1 \mathrm{H})$, $6.05(\mathrm{~s}, 1 \mathrm{H}), 6.02(\mathrm{~s}, 1 \mathrm{H}), 5.18(\mathrm{~d}, J=14.8 \mathrm{~Hz}, 1 \mathrm{H}), 5.04(\mathrm{~d}, J=15.6 \mathrm{~Hz}, 1 \mathrm{H}), 2.3(\mathrm{~s}, 3 \mathrm{H}) .{ }^{13} \mathrm{C}-\mathrm{NMR}$ (100 MHz, CDCl3) $\delta 140.20,138.73,137.93,136.31,132.18,129.71,128.98,128.91,128.85,127.94$, $127.48,127.08,126.30,126.23,125.59,24.60,124.37,114.87,106.80,85.08,67.27,21.42$. LRMS (EI) $\mathrm{m} / z 403\left(\mathrm{M}^{+}\right)$; HRMS (EI) $\mathrm{m} / z$ calcd $\mathrm{C}_{23} \mathrm{H}_{18} \mathrm{BrNO}\left(\mathrm{M}^{+}\right)$403.0572, found 403.0576.

3-Fluoro-12-phenyl-4b,6-dihydrobenzo[4,5][1,3]oxazino[2,3-a]isoquinoline (3Fa). ${ }^{1} \mathrm{H}-\mathrm{NMR}$ (400 MHz, $\left.\mathrm{CDCl}_{3}\right) \delta 7.29-7.27(\mathrm{~m}, 5 \mathrm{H}), 7.20-7.16(\mathrm{~m}, 2 \mathrm{H}), 7.10-7.02(\mathrm{~m}, 2 \mathrm{H}), 6.93(\mathrm{t}, J=7.6 \mathrm{~Hz}, 1 \mathrm{H}), 6.82(\mathrm{t}$, $J=7.6 \mathrm{~Hz}, 1 \mathrm{H}), 6.24(\mathrm{~d}, J=8.0 \mathrm{~Hz}, 1 \mathrm{H}), 6.09(\mathrm{~s}, 1 \mathrm{H}), 6.04(\mathrm{~s}, 1 \mathrm{H}), 5.24(\mathrm{~d}, J=14.4 \mathrm{~Hz}, 1 \mathrm{H}), 5.10(\mathrm{~d}$, $J=14.4 \mathrm{~Hz}, 1 \mathrm{H}) .{ }^{13} \mathrm{C}-\mathrm{NMR}\left(100 \mathrm{MHz}, \mathrm{CDCl}_{3}\right) \delta 161.49(\mathrm{~d}, J=243.5 \mathrm{~Hz}), 140.01,139.38,136.64$, $129.23,129.13(\mathrm{~d}, J=7.6 \mathrm{~Hz}), 128.73,128.38,128.11,127.99,126.13,125.86$ (d, $J=7.9 \mathrm{~Hz})$, 124.70, 123.18, 122.24, 116.09 (d, $J=22.0 \mathrm{~Hz}), 113.16$ (d, $J=22.6 \mathrm{~Hz}), 105.86,84.67,67.86$. LRMS (EI) $\mathrm{m} / z 329\left(\mathrm{M}^{+}\right)$; HRMS (EI) $\mathrm{m} / z$ calcd $\mathrm{C}_{22} \mathrm{H}_{16} \mathrm{FNO}\left(\mathrm{M}^{+}\right) 329.1216$, found 329.1210.

8-Chloro-3-fluoro-12-phenyl-4b,6-dihydrobenzo[4,5][1,3] oxazino[2,3-a] isoquinoline (3Fc). ${ }^{1} \mathrm{H}-\mathrm{NMR}$ $\left(400 \mathrm{MHz}, \mathrm{CDCl}_{3}\right) \delta 7.30-7.23(\mathrm{~m}, 5 \mathrm{H}), 7.18-7.13(\mathrm{~m}, 2 \mathrm{H}), 7.06-7.00(\mathrm{~m}, 2 \mathrm{H}), 6.76$ (dd, J=8.8, 2.4 Hz, $1 \mathrm{H}), 6.11(\mathrm{~d}, J=8.8 \mathrm{~Hz}, 1 \mathrm{H}), 6.06(\mathrm{~s}, 1 \mathrm{H}), 6.02(\mathrm{~s}, 1 \mathrm{H}), 5.14(\mathrm{~d}, J=14.4 \mathrm{~Hz}, 1 \mathrm{H}), 5.02(\mathrm{~d}, J=14.4 \mathrm{~Hz}$, 1H). ${ }^{13} \mathrm{C}-\mathrm{NMR}(100 \mathrm{MHz}, \mathrm{CDCl} 3) \delta 162.80(\mathrm{~d}, J=244.2 \mathrm{~Hz}), 139.40,137.77,136.13,129.25(\mathrm{~d}$, $J=7.5 \mathrm{~Hz}), 129.06,128.48,128.45,128.28,128.22,127.20,126.34,125.98$ (d, $J=7.7 \mathrm{~Hz}), 124.64$, 123.85, $116.12(\mathrm{~d}, J=21.9 \mathrm{~Hz}), 112.96(\mathrm{~d}, J=22.9 \mathrm{~Hz}), 106.75,84.71,67.25$. LRMS (EI) $\mathrm{m} / z 363$ $\left(\mathrm{M}^{+}\right)$; HRMS (EI) $m / z$ calcd $\mathrm{C}_{22} \mathrm{H}_{15} \mathrm{ClFNO}\left(\mathrm{M}^{+}\right)$363.0826, found 363.0808 .

8-Bromo-3-fluoro-12-phenyl-4b,6-dihydrobenzo[4,5][1,3] oxazino[2,3-a]isoquinoline (3Fe). ${ }^{1} \mathrm{H}-\mathrm{NMR}$ $\left(400 \mathrm{MHz} \mathrm{CDCl}_{3}\right) \delta 7.30-7.24(\mathrm{~m}, 5 \mathrm{H}), 7.20(\mathrm{~d}, J=2.4 \mathrm{~Hz}, 1 \mathrm{H}), 7.17-7.13(\mathrm{~m}, 2 \mathrm{H}), 7.04(\mathrm{td}, J=8.4$, $2.4 \mathrm{~Hz}, 1 \mathrm{H}), 6.90(\mathrm{dd}, J=8.8,2.4 \mathrm{~Hz}, 1 \mathrm{H}), 6.09(\mathrm{~s}, 1 \mathrm{H}), 6.05(\mathrm{~d}, J=8.8 \mathrm{~Hz}, 1 \mathrm{H}), 6.02(\mathrm{~s}, 1 \mathrm{H}), 5.13(\mathrm{~d}$, $J=14.0 \mathrm{~Hz}, 1 \mathrm{H}), 5.08-4.96(\mathrm{~d}, J=14.4 \mathrm{~Hz}, 1 \mathrm{H}) .{ }^{13} \mathrm{C}-\mathrm{NMR}\left(100 \mathrm{MHz}, \mathrm{CDCl}_{3}\right) \delta 161.64(\mathrm{~d}, J=244.1 \mathrm{~Hz})$, 139.25, 138. 19, 136.06, $129.46(\mathrm{~d}, J=7.2 \mathrm{~Hz}), 129.38,129.26,128.42,128.32,128.22,128.16$, $127.54,125.98$ (d, $J=7.9 \mathrm{~Hz}), 124.02,116.07(\mathrm{~d}, J=22.0 \mathrm{~Hz}), 114.62,112.85(\mathrm{~d}, J=23.0 \mathrm{~Hz})$, 107.12, 84.71, 67.10. LRMS (EI) $\mathrm{m} / z 407\left(\mathrm{M}^{+}\right)$; HRMS (EI) $m / z$ calcd $\mathrm{C}_{22} \mathrm{H}_{15} \mathrm{BrFNO}\left(\mathrm{M}^{+}\right)$407.0321, found 407.0316 .

3-Fluoro-8-methyl-12-phenyl-4b,6-dihydrobenzo[4,5][1,3]oxazino[2,3-a]isoquinoline (3Ff). ${ }^{1} \mathrm{H}-\mathrm{NMR}$ $\left(400 \mathrm{MHz}, \mathrm{CDCl}_{3}\right) \delta 7.26-7.19(\mathrm{~m}, 5 \mathrm{H}), 7.16-7.11(\mathrm{~m}, 2 \mathrm{H}), 7.02(\mathrm{td}, J=8.4,2.4 \mathrm{~Hz}, 1 \mathrm{H}), 6.86(\mathrm{~s}$, $1 \mathrm{H}), 6.60(\mathrm{~d}, J=8.4 \mathrm{~Hz}, 1 \mathrm{H}), 6.11(\mathrm{~d}, J=8.4 \mathrm{~Hz}, 1 \mathrm{H}), 6.03(\mathrm{~s}, 1 \mathrm{H}), 5.91(\mathrm{~s}, 1 \mathrm{H}), 5.21(\mathrm{~d}, J=14.8 \mathrm{~Hz}$, $1 \mathrm{H}), 5.05(\mathrm{~d}, J=14.8 \mathrm{~Hz}, 1 \mathrm{H}), 2.23(\mathrm{~s}, 3 \mathrm{H}) .{ }^{13} \mathrm{C}-\mathrm{NMR}(100 \mathrm{MHz}, \mathrm{CDCl} 3) \delta 160.78(\mathrm{~d}, J=243.8 \mathrm{~Hz})$, $140.30,137.07,136.80,132.22,128.86,128.74,128.54,128.29$ (d, $J=7.8 \mathrm{~Hz}), 127.97,127.84$, 126.66, 125.77 (d, $J=7.7 \mathrm{~Hz}), 125.10,123.50,116.18$ (d, $J=22.8 \mathrm{~Hz}), 113.48(\mathrm{~d}, J=22.3 \mathrm{~Hz})$, 104.42, 84.59, 67.94, 20.80. LRMS (EI) $\mathrm{m} / z 343\left(\mathrm{M}^{+}\right)$; HRMS (EI) $\mathrm{m} / z$ calcd $\mathrm{C}_{23} \mathrm{H}_{18} \mathrm{FNO}\left(\mathrm{M}^{+}\right.$) 343.1372 , found 343.1375 . 
2-Methyl-12-phenyl-4b,6-dihydrobenzo[4,5][1,3]oxazino[2,3-a]isoquinoline (3Ga). ${ }^{1} \mathrm{H}-\mathrm{NMR}$ (400 MHz, $\left.\mathrm{CDCl}_{3}\right) \delta 7.31(\mathrm{~d}, J=8.0 \mathrm{~Hz}, 1 \mathrm{H}), 7.26-7.19(\mathrm{~m}, 5 \mathrm{H}), 7.07-7.03(\mathrm{~m}, 2 \mathrm{H}), 7.00(\mathrm{~s}, 1 \mathrm{H}), 6.92(\mathrm{t}$, $J=7.6 \mathrm{~Hz}, 1 \mathrm{H}), 6.78(\mathrm{t}, J=7.6 \mathrm{~Hz}, 1 \mathrm{H}), 6.22(\mathrm{~d}, J=8.4 \mathrm{~Hz}, 1 \mathrm{H}), 6.07(\mathrm{~s}, 1 \mathrm{H}), 5.90(\mathrm{~s}, 1 \mathrm{H}), 5.26(\mathrm{~d}$, $J=14.8 \mathrm{~Hz}, 1 \mathrm{H}), 5.09$ (d, $J=14.4 \mathrm{~Hz}, 1 \mathrm{H}), 2.36(\mathrm{~s}, 3 \mathrm{H}) .{ }^{13} \mathrm{C}-\mathrm{NMR}\left(125 \mathrm{MHz}, \mathrm{CDCl}_{3}\right) \delta 140.81,140.05$, $138.74,137.11,132.24,128.65,128.52,127.97,127.79,126.94,126.78,125.69,124.79,124.70$, 124.12, 123.88, 122.54, 105.59, 85.00, 68.00, 21.40. LRMS (EI) $m / z 325\left(\mathrm{M}^{+}\right)$; HRMS (EI) $\mathrm{m} / z$ calcd $\mathrm{C}_{23} \mathrm{H}_{19} \mathrm{NO}\left(\mathrm{M}^{+}\right)$325.1467, found 325.1463

8-Chloro-2-methyl-12-phenyl-4b,6-dihydrobenzo[4,5][1,3]oxazino[2,3-a]isoquinoline (3Gc). ${ }^{1} \mathrm{H}-\mathrm{NMR}$ $\left(400 \mathrm{MHz}, \mathrm{CDCl}_{3}\right) \delta 7.32-7.25(\mathrm{~m}, 4 \mathrm{H}), 7.23-7.21(\mathrm{~m}, 2 \mathrm{H}), 7.08(\mathrm{~d}, J=8.0 \mathrm{~Hz}, 1 \mathrm{H}), 7.04(\mathrm{~d}, J=1.6 \mathrm{~Hz}$, $1 \mathrm{H}), 7.01(\mathrm{~s}, 1 \mathrm{H}), 6.75(\mathrm{dd}, J=8.8,2.4 \mathrm{~Hz}, 1 \mathrm{H}), 6.13(\mathrm{~d}, J=8.8 \mathrm{~Hz}, 1 \mathrm{H}), 6.02(\mathrm{~s}, 1 \mathrm{H}), 5.95(\mathrm{~s}, 1 \mathrm{H})$, $5.18(\mathrm{~d}, J=14.8 \mathrm{~Hz}, 1 \mathrm{H}), 5.04(\mathrm{~d}, J=14.8 \mathrm{~Hz}, 1 \mathrm{H}), 2.34(\mathrm{~s}, 3 \mathrm{H}) .{ }^{13} \mathrm{C}-\mathrm{NMR}\left(125 \mathrm{MHz}, \mathrm{CDCl}_{3}\right) \delta 140.27$, $138.87,138.51,136.68,132.07,129.69,128.56,128.21,128.05,127.60,127.21,126.58,125.99$, 124.95, 124.67, 124.23, 106.49, 85.14, 67.49, 21.41. LRMS (EI) $m / z 359\left(\mathrm{M}^{+}\right)$; HRMS (EI) $m / z$ calcd $\mathrm{C}_{23} \mathrm{H}_{18} \mathrm{ClNO}\left(\mathrm{M}^{+}\right) 359.1077$, found 359.1058.

2,8-Dimethyl-12-phenyl-4b,6-dihydrobenzo[4,5][1,3]oxazino[2,3-a]isoquinoline $\quad$ (3Gf). $\quad{ }^{1} \mathrm{H}-\mathrm{NMR}$ $\left(400 \mathrm{MHz}, \mathrm{CDCl}_{3}\right) \delta 7.31-7.28(\mathrm{~d}, J=8.0 \mathrm{~Hz}, 1 \mathrm{H}), 7.26-7.18(\mathrm{~m}, 5 \mathrm{H}), 7.04(\mathrm{~d}, J=7.6 \mathrm{~Hz}, 1 \mathrm{H}), 6.99$ $(\mathrm{s}, 1 \mathrm{H}), 6.85(\mathrm{~s}, 1 \mathrm{H}), 6.59(\mathrm{~d}, J=7.6 \mathrm{~Hz}, 1 \mathrm{H}), 6.12(\mathrm{~d}, J=8.0 \mathrm{~Hz}, 1 \mathrm{H}), 6.04(\mathrm{~s}, 1 \mathrm{H}), 5.81(\mathrm{~s}, 1 \mathrm{H}), 5.25$ $(\mathrm{d}, J=14.8 \mathrm{~Hz}, 1 \mathrm{H}), 5.06(\mathrm{~d}, J=14.8 \mathrm{~Hz}, 1 \mathrm{H}), 2.36(\mathrm{~s}, 3 \mathrm{H}), 2.24(\mathrm{~s}, 3 \mathrm{H}) .{ }^{13} \mathrm{C}-\mathrm{NMR}\left(125 \mathrm{MHz}, \mathrm{CDCl}_{3}\right)$ $\delta 141.11,138.76,137.73,137.30,132.43,132.29,128.82,128.52,127.89,127.69,127.14,126.73$, 126.36, 125.10, 124.69, 124.08, 123.59, 104.46, 84.99, 68.06, 21.42, 20.87. LRMS (EI) m/z $339\left(\mathrm{M}^{+}\right)$; HRMS (EI) $m / z$ calcd $\mathrm{C}_{24} \mathrm{H}_{21} \mathrm{NO}\left(\mathrm{M}^{+}\right)$339.1623, found 339.1617.

2-Methyl-14-phenyl-4b,6-dihydronaphtho[2',3':4,5][1,3]oxazino[2,3-a]isoquinoline (3Gh). ${ }^{1} \mathrm{H}-\mathrm{NMR}$ $\left(400 \mathrm{MHz} \mathrm{CDCl}_{3}\right) \delta 7.68(\mathrm{~d}, J=8.4 \mathrm{~Hz}, 1 \mathrm{H}), 7.54(\mathrm{~s}, 1 \mathrm{H}), 7.42-7.36(\mathrm{~m}, 3 \mathrm{H}), 7.27-7.25(\mathrm{~m}, 6 \mathrm{H}), 7.10$ $(\mathrm{d}, J=7.6 \mathrm{~Hz}, 1 \mathrm{H}), 7.07(\mathrm{~s}, 1 \mathrm{H}), 6.51(\mathrm{~s}, 1 \mathrm{H}), 6.31(\mathrm{~s}, 1 \mathrm{H}), 6.21(\mathrm{~s}, 1 \mathrm{H}), 5.28(\mathrm{~d}, J=13.6 \mathrm{~Hz}, 1 \mathrm{H})$, $5.22(\mathrm{~d}, J=14.0 \mathrm{~Hz}, 1 \mathrm{H}), 2.39(\mathrm{~s}, 3 \mathrm{H}) .{ }^{13} \mathrm{C}-\mathrm{NMR}\left(125 \mathrm{MHz}, \mathrm{CDCl}_{3}\right) \delta 140.27,138.34,137.00$, $136.43,132.78,132.32,128.77,128.33,128.17,128.10,128.03,127.60,127.20,126.88,126.53$, $125.76,124.98,124.87,124.12,123.19,118.14,109.99,85.88,67.63,21.39$. LRMS (EI) $\mathrm{m} / z 375$ $\left(\mathrm{M}^{+}\right)$; HRMS (EI) $m / z$ calcd $\mathrm{C}_{27} \mathrm{H}_{21} \mathrm{NO}\left(\mathrm{M}^{+}\right) 375.1623$, found 375.1617 .

12-Hexyl-4b, 6-dihydrobenzo[4,5] [1,3]oxazino[2,3-a]isoquinoline (3Ha). ${ }^{1} \mathrm{H}-\mathrm{NMR}\left(400 \mathrm{MHz}, \mathrm{CDCl}_{3}\right)$ $\delta 7.35-7.31(\mathrm{~m}, 3 \mathrm{H}), 7.27-7.09(\mathrm{~m}, 5 \mathrm{H}), 5.83(\mathrm{~s}, 1 \mathrm{H}), 5.75(\mathrm{~s}, 1 \mathrm{H}), 5.23(\mathrm{~d}, J=15.6 \mathrm{~Hz}, 1 \mathrm{H}), 5.00(\mathrm{~d}$, $J=15.2 \mathrm{~Hz}, 1 \mathrm{H}), 2.57-2.36(\mathrm{~m}, 2 \mathrm{H}), 1.46-1.31(\mathrm{~m}, 2 \mathrm{H}), 1.25-1.13(\mathrm{~m}, 6 \mathrm{H}), 0.85(\mathrm{t}, J=6.4 \mathrm{~Hz}, 3 \mathrm{H})$.

${ }^{13} \mathrm{C}-\mathrm{NMR}\left(100 \mathrm{MHz}, \mathrm{CDCl}_{3}\right) \delta 142.63,140.91,132.49,130.91,129.23,127.63,125.91,125.31$, $125.15,125.00,124.92,124.85,123.62,100.42,84.85,67.74,32.88,31.52,28.75,27.57,22.43,14.02$. LRMS (EI) $m / z 319\left(\mathrm{M}^{+}\right)$; HRMS (EI) $m / z$ calcd $\mathrm{C}_{22} \mathrm{H}_{25} \mathrm{NO}\left(\mathrm{M}^{+}\right)$319.1936, found 319.1935.

12-Hexyl-8-iodo-4b,6-dihydrobenzo[4,5][1,3] oxa-zino[2,3-a]isoquinoline (3Hi). ${ }^{1} \mathrm{H}-\mathrm{NMR}$ (300 MHz, $\left.\mathrm{CDCl}_{3}\right) \delta 7.54(\mathrm{~d}, J=8.7 \mathrm{~Hz}, 1 \mathrm{H}), 7.43(\mathrm{~s}, 1 \mathrm{H}), 7.34-7.27(\mathrm{~m}, 2 \mathrm{H}), 7.19-7.10(\mathrm{~m}, 2 \mathrm{H}), 6.92(\mathrm{~d}, J=8.4 \mathrm{~Hz}$, $1 \mathrm{H}), 5.74(\mathrm{~s}, 2 \mathrm{H}), 5.14(\mathrm{~d}, J=15.3 \mathrm{~Hz}, 1 \mathrm{H}), 4.91(\mathrm{~d}, J=15.9 \mathrm{~Hz}, 1 \mathrm{H}), 2.43-2.40(\mathrm{~m}, 2 \mathrm{H}), 1.30-1.17$ $(\mathrm{m}, 8 \mathrm{H}), 0.88-0.84(\mathrm{~m}, 3 \mathrm{H}) .{ }^{13} \mathrm{C}-\mathrm{NMR}\left(125 \mathrm{MHz}, \mathrm{CDCl}_{3}\right) \delta 141.92,140.66,134.94,134.27,133.07$, 
132.26, 129.39, 127.60, 126.78, 125.19, 124.88, 123.77, 101.09, 84.83, 67.02, 32.73, 31.51, 28.74, 27.59, 22.46, 14.07. LRMS (EI) $m / z 445\left(\mathrm{M}^{+}\right.$); HRMS (EI) $m / z$ calcd $\mathrm{C}_{22} \mathrm{H}_{24} \mathrm{INO}\left(\mathrm{M}^{+}\right)$445.0903, found 445.0905 .

6-Phenyl-11b, 13-dihydropyrido[2',3':4,5] [1,3]oxa-zino[2,3-a] isoquinoline (3Aj). ${ }^{1} \mathrm{H}-\mathrm{NMR}(500 \mathrm{MHz}$, $\left.\mathrm{CDCl}_{3}\right) \delta 7.81(\mathrm{~d}, J=5.5 \mathrm{~Hz}, 1 \mathrm{H}), 7.46(\mathrm{~d}, J=7.5 \mathrm{~Hz}, 1 \mathrm{H}), 7.36-7.28(\mathrm{~m}, 6 \mathrm{H}), 7.26-7.19(\mathrm{~m}, 3 \mathrm{H})$, $6.71(\mathrm{~m}, 1 \mathrm{H}), 6.34(\mathrm{~s}, 1 \mathrm{H}), 6.23(\mathrm{~s}, 1 \mathrm{H}), 5.09(\mathrm{~d}, J=14.0 \mathrm{~Hz}, 1 \mathrm{H}), 5.03(\mathrm{~d}, J=13.5 \mathrm{~Hz}, 1 \mathrm{H}) .{ }^{13} \mathrm{C}-\mathrm{NMR}$ $\left(125 \mathrm{MHz}, \mathrm{CDCl}_{3}\right) \delta 150.45,146.85,139.24,137.51,132.44,132.28,129.63,128.69,128.04,127.49$, 127.06, 126.87, 124.91, 124.52, 121.24, 116.31, 111.25, 85.88, 66.45. LRMS (EI) $\mathrm{m} / z 312\left(\mathrm{M}^{+}\right)$; HRMS (EI) $m / z$ calcd $\mathrm{C}_{21} \mathrm{H}_{16} \mathrm{~N}_{2} \mathrm{O}\left(\mathrm{M}^{+}\right) 312.1263$, found 312.1260 .

6-(4-Fluorophenyl)-11b,13-dihydropyrido[2',3':4,5][1,3]oxazino[2,3-a]isoquinoline (3Bj). ${ }^{1} \mathrm{H}-\mathrm{NMR}$ $\left(400 \mathrm{MHz}, \mathrm{CDCl}_{3}\right) \delta 7.81(\mathrm{dd}, J=4.8,1.6 \mathrm{~Hz}, 1 \mathrm{H}), 7.44(\mathrm{~d}, J=7.2 \mathrm{~Hz}, 1 \mathrm{H}), 7.34-7.26(\mathrm{~m}, 4 \mathrm{H})$, $7.25-7.22(\mathrm{~m}, 1 \mathrm{H}), 7.18(\mathrm{dd}, J=7.6,1.2 \mathrm{~Hz}, 1 \mathrm{H}), 6.94(\mathrm{t}, J=8.8 \mathrm{~Hz}, 2 \mathrm{H}), 6.74-6.70(\mathrm{q}, 1 \mathrm{H}), 6.26(\mathrm{~s}$, $1 \mathrm{H}), 6.20(\mathrm{~s}, 1 \mathrm{H}), 5.06(\mathrm{~d}, J=14.0 \mathrm{~Hz}, 1 \mathrm{H}), 5.04-4.98(\mathrm{~d}, J=14.0 \mathrm{~Hz}, 1 \mathrm{H}) .{ }^{13} \mathrm{C}-\mathrm{NMR}(100 \mathrm{MHz}$, $\left.\mathrm{CDCl}_{3}\right) \delta 150.19,146.75,138.22,133.53,132.39,132.20,129.38,128.72,128.65,126.89,124.70(\mathrm{~d}$, $J=25.6 \mathrm{~Hz}), 121.32,116.46,114.94(\mathrm{~d}, J=21.5 \mathrm{~Hz}), 110.90,85.78,66.37$. LRMS (EI) $\mathrm{m} / z 330\left(\mathrm{M}^{+}\right)$; HRMS (EI) $m / z$ calcd ${ }_{\mathrm{C} 21} \mathrm{H}_{15} \mathrm{FN}_{2} \mathrm{O}\left(\mathrm{M}^{+}\right) 330.1168$, found 330.1162 .

\section{Conclusions}

In summary, an efficient strategy for the one-pot construction of dihydrobenzo[4,5][1,3]oxazino[2,3-a]isoquinolines from simple starting substrates has been developed via an unprecedented $\mathrm{Ag}(\mathrm{I})$-catalyzed $\mathrm{N}$-aryliminium ion cyclization cascade. On the basis of a large assortment of bioactivities of fused isoquinolines, we believe that the new synthetic method will serve as valuable tool to efficiently construct new members fused isoquinoline derivatives in drug discovery, and will have potential biological applications.

\section{Supplementary Materials}

Supplementary materials can be accessed at: http://www.mdpi.com/1420-3049/18/1/814/s1.

\section{Acknowledgments}

We gratefully acknowledge financial support from the National Natural Science Foundation of China (Grants 20902022, 81102307, 21222211), the Natural Science Foundation of Shanghai, China (Grant 11ZR1444600), National S\&T Major Project, China (Grant 2011ZX09102-005-02), the Shanghai Committee of Science and Technology (Grant 11DZ2260600), and the Fundamental Research Funds for the Central Universities.

\section{References}

1. Parenty, A.D.; Smith, L.V.; Guthrie, K.M.; Long, D.L.; Plumb, J.; Brown, R.; Cronin, L. Highly stable phenanthridinium frameworks as a new class of tunable DNA binding agents with cytotoxic properties. J. Med. Chem. 2005, 48, 4504-4506. 
2. Weinkauf, R.L.; Chen, A.Y.; Yu, C.; Liu, L.; Barrows, L.; LaVoie, E.J. Antineoplastic activity of benzimidazo[1,2-b]-isoquinolines, indolo[2,3-b]quinolines, and pyridocarbazoles. Bioorg. Med. Chem. 1994, 2, 781-786.

3. Chen, Z.; Wu, J. Efficient generation of biologically active H-pyrazolo[5,1-a]isoquinolines via multicomponent reaction. Org. Lett. 2010, 12, 4856-4859.

4. Iwasa, K.; Moriyasu, M.; Tachibana, Y.; Kim, H.S.; Wataya, Y.; Wiegrebe, W.; Bastow, K.F.; Cosentino, L.M.; Kozuka, M.; Lee, K.H. Simple isoquinoline and benzylisoquinoline alkaloids as potential antimicrobial, antimalarial, cytotoxic, and anti-HIV agents. Bioorg. Med. Chem. 2001, 9, 2871-2884.

5. Reddy, M.V.; Rao, M.R.; Rhodes, D.; Hansen, M.S.; Rubins, K.; Bushman, F.D.; Venkateswarlu, Y.; Faulkner, D.J. Lamellarin alpha 20-sulfate, an inhibitor of HIV-1 integrase active against HIV-1 virus in cell culture. J. Med. Chem. 1999, 42, 1901-1907.

6. Petrignet, J.; Boudhar, A.; Blond, G.; Suffert, J. Step-economical synthesis of taxol-like tricycles through a palladium-catalyzed domino reaction. Angew. Chem. Int. Ed. 2011, 50, 3285-3289.

7. Chen, J.R.; Li, C.F.; An, X.L.; Zhang, J.J.; Zhu, X.Y.; Xiao, W.J. Ru-catalyzed tandem crossmetathesis/intramolecular-hydroarylation sequence. Angew. Chem. Int. Ed. 2008, 47, 2489-2492.

8. Wasilke, J.C.; Obrey, S.J.; Baker, R.T.; Bazan, G.C. Concurrent tandem catalysis. Chem. Rev. 2005, 105, 1001-1020.

9. Velcicky, J.; Soicke, A.; Steiner, R.; Schmalz, H.G. Palladium-catalyzed cyanomethylation of aryl halides through domino Suzuki coupling-isoxazole fragmentation. J. Am. Chem. Soc. 2011, 133, 6948-6951.

10. Malakar, C.C.; Schmidt, D.; Conrad, J.; Beifuss, U. Cu(I)-catalyzed domino reactions: efficient and selective synthesis of 4H-chromenes and naphthalenes. Org. Lett. 2011, 13, 1972-1975.

11. Nanjo, T.; Tsukano, C.; Takemoto, Y. Palladium-catalyzed cascade process consisting of isocyanide insertion and benzylic $\mathrm{C}(\mathrm{sp} 3)-\mathrm{H}$ activation: concise synthesis of indole derivatives. Org. Lett. 2012, 14, 4270-4273.

12. Magano, J.; Dunetz, J.R. Large-scale applications of transition metal-catalyzed couplings for the synthesis of pharmaceuticals. Chem. Rev. 2011, 111, 2177-2250.

13. Kumar, K.S.; Kumar, P.M.; Reddy, M.A.; Ferozuddin, M.; Sreenivasulu, M.; Jafar, A.A.; Krishna, G.R.; Reddy, C.M.; Rambabu, D.; Kumar, K.S.; et al. Yb(OTf)3 catalyzed new cascade reaction: A facile assembly of fused quinazolinones. Chem. Commun. 2011, 47, 10263-10265.

14. Gigant, N.; Gillaizeau, I. Palladium(II)-catalyzed direct alkenylation of nonaromatic enamides. Org. Lett. 2012, 14, 3304-3307.

15. Patil, N.T.; Mutyala, A.K.; Konala, A.; Tella, R.B. Tuning the reactivity of Au-complexes in an $\mathrm{Au}(\mathrm{I}) /$ chiral Bronsted acid cooperative catalytic system: an approach to optically active fused 1,2-dihydroisoquinolines. Chem. Commun. 2012, 48, 3094-3096.

16. Ouyang, H.C.; Tang, R.Y.; Zhong, P.; Zhang, X.G.; Li, J.H. CuI/I2-promoted electrophilic tandem cyclization of 2-ethynylbenzaldehydes with ortho-benzenediamines: Synthesis of iodoisoquinoline-fused benzimidazoles. J. Org. Chem. 2011, 76, 223-228. 
17. Patil, N.T.; Mutyala, A.K.; Lakshmi, P.G.V.V.; Raju, P.V.K.; Sridhar, B. Facile Assembly of Fused Isoquinolines by Gold(I)-Catalyzed-Coupling Cyclization Reactions between o-Alkynylbenzaldehydes and Aromatic Amines Containing Tethered Nucleophiles. Eur. J. Org. Chem. 2010, 1999-2007.

18. Yanada, R.; Hashimoto, K.; Tokizane, R.; Miwa, Y.; Minami, H.; Yanada, K.; Ishikura, M.; Takemoto, Y. Indium(III)-catalyzed tandem reaction with alkynylbenzaldehydes and alkynylanilines to heteroaromatic compounds. J. Org. Chem. 2008, 73, 5135-5138.

19. Yanada, R.; Obika, S.; Kono, H.; Takemoto, Y. In(OTf)3-catalyzed tandem nucleophilic addition and cyclization of ortho-alkynylarylaldimines to 1,2-dihydroisoquinolines. Angew. Chem. Int. Ed. 2006, 45, 3822-3825.

20. Liu, G.; Zhou, Y.; Ye, D.; Zhang, D.; Ding, X.; Jiang, H.; Liu, H. Silver-Catalyzed Intramolecular Cyclization of o-(1-Alkynyl)benzamides: Efficient Synthesis of (1H)-Isochromen-1-imines. Adv. Synth. Catal. 2009, 351, 2605-2610.

21. Patil, N.T.; Pahadi, N.K.; Yamamoto, Y. Silver(I)-catalyzed novel cascade cyclization reactions: incorporation of allenes into the isochromenes. J. Org. Chem. 2005, 70, 10096-10098.

22. Blanc, A.; Tenbrink, K.; Weibel, J.M.; Pale, P. Silver(I)-catalyzed cascade: Direct access to furans from alkynyloxiranes. J. Org. Chem. 2009, 74, 4360-4363.

23. Su, S.; Porco, J.A., Jr. Synthesis of pyrrolo-isoquinolines related to the lamellarins using silvercatalyzed cycloisomerization/dipolar cycloaddition. J. Am. Chem. Soc. 2007, 129, 7744-7745.

24. Weibel, J.M.; Blanc, A.; Pale, P. Ag-mediated reactions: Coupling and heterocyclization reactions. Chem. Rev. 2008, 108, 3149-3173.

25. Huple, D.B.; Chen, C.H.; Das, A.; Liu, R.S. Silver-catalyzed exo-dig-azacyclization/[3+2] cycloaddition cascades on 1-tosylhydrazon-4-oxy-5-yne substrates: Applicability to diverse alkenes. Adv. Synth. Catal. 2011, 353, 1877-1882.

26. Li, W.; Wang, Y.; Lu, T. Facile synthesis of 1-aminoisoquinolines via the tandem reactions of 2-alkynylbenzaldoximes with isothiocyanates. Tetrahedron 2012, 68, 6843-6848.

27. Huang, P.; Yang, Q.; Chen, Z.; Ding, Q.; Xu, J.; Peng, Y. Metal cocatalyzed tandem alkynylative cyclization reaction of in situ formed $\mathrm{N}$-iminoisoquinolinium ylides with bromoalkynes via $\mathrm{C}-\mathrm{H}$ bond activation. J. Org. Chem. 2012, 77, 8092-8098.

28. Rustagi, V.; Aggarwal, T.; Verma, A.K. Highly efficient Ag(I)-catalyzed regioselective tandem synthesis of diversely substituted quinoxalines and benzimidazoles in water. Green Chem. 2011, $13,1640-1643$.

29. Rustagi, V.; Tiwari, R.; Verma, A.K. AgI-Catalyzed Cascade Strategy: Regioselective Access to Diversely Substituted Fused Benzimidazo[2,1-a]isoquinolines, Naphthyridines, Thienopyridines, and Quinoxalines in Water. Eur. J. Org. Chem. 2012, 4590-4602.

30. Yu, X.X.; Yang, Q.; Lou, H.L.; Peng, Y.Y.; Wu, J. An efficient approach to pyrazolo[5,1-a]isoquinolin-2-amines via a silver(I)-catalyzed three-component reaction of 2-alkynylbenzaldehyde, sulfonohydrazide, and nitrile. Org. Biomol. Chem. 2011, 9, 7033-7037.

31. Markina, N.A.; Mancuso, R.; Neuenswander, B.; Lushington, G.H.; Larock, R.C. Solution-Phase Parallel Synthesis of a Diverse Library of 1,2-Dihydroisoquinolines. ACS Comb. Sci. 2011, 13, 265-271. 
32. Ding, Q.P.; Wu, J. Lewis acid- and organocatalyst-cocatalyzed multicomponent reactions of 2-alkynylbenzaldehydes, amines, and ketones. Org. Lett. 2007, 9, 4959-4962.

33. Yu, X.X.; Wu, J. Synthesis of 1-(1H-Indol-3-yl)-1,2-dihydroisoquinolines via AgOTf-Catalyzed Three-Component Reactions of 2-Alkynylbenzaldehydes, Amines, and Indoles. J. Comb. Chem. 2010, 12, 238-244.

34. Asao, N.; Yudha, S.S.; Nogami, T.; Yamamoto, Y. Direct Mannich and nitro-Mannich reactions with non-activated imines: AgOTf-catalyzed addition of pronucleophiles to ortho-alkynylaryl aldimines leading to 1,2-dihydroisoquinolines. Angew. Chem. Int. Ed. 2005, 44, 5526-5528.

35. Liu, G.N.; Zhou, Y.; Lin, D.Z.; Wang, J.F.; Zhang, L.; Jiang, H.L.; Liu, H. Synthesis of Pyrrolo[1,2-a]quinoxalines via Gold(I)-Mediated Cascade Reactions. ACS Comb. Sci. 2011, 13, 209-213.

36. Zhou, Y.; Li, J.; Ji, X.; Zhou, W.; Zhang, X.; Qian, W.; Jiang, H.; Liu, H. Silver- and gold-mediated domino transformation: A strategy for synthesizing benzo[e]indolo[1,2a]pyrrolo/pyrido[2,1-c][1,4]diazepine-3,9-diones. J. Org. Chem. 2011, 76, 1239-1249.

37. Feng, E.; Zhou, Y.; Zhang, D.; Zhang, L.; Sun, H.; Jiang, H.; Liu, H. Gold(I)-catalyzed tandem transformation: A simple approach for the synthesis of pyrrolo/pyrido[2,1-a][1,3]benzoxazinones and pyrrolo/pyrido[2,1-a]quinazolinones. J. Org. Chem. 2010, 75, 3274-3282.

38. Feng, E.G.; Zhou, Y.; Zhao, F.; Chen, X.J.; Zhang, L.; Jiang, H.L.; Liu, H. Gold-catalyzed tandem reaction in water: An efficient and convenient synthesis of fused polycyclic indoles. Green Chem. 2012, 14, 1888-1895.

39. Zhou, Y.; Feng, E.G.; Liu, G.N.; Ye, D.J.; Li, J.; Jiang, H.L.; Liu, H., Liu, G. Gold-Catalyzed One-Pot Cascade Construction of Highly Functionalized Pyrrolo[1,2-a]quinolin-1(2H)-ones. J. Org. Chem. 2009, 74, 7344-7348.

40. Zhou, Y.; Zhai, Y.; Ji, X.; Liu, G.N.; Feng, E.G.; Ye, D.J.; Zhao, L.X.; Jiang, H.L.; Liu, H. Gold(I)-Catalyzed One-Pot Tandem Coupling/Cyclization: An Efficient Synthesis of Pyrrolo/Pyrido[2,1-b]benzo[d][1,3]oxazin-1-ones. Adv. Synth. Catal. 2010, 352, 373-378.

41. Zhou, Y.; Ji, X.; Liu, G.N.; Zhang, D.Y.; Zhao, L.X.; Jiang, H.L.; Liu, H. Gold(I)-Catalyzed Cascade for Synthesis of Pyrrolo[1,2-a:2',1'-c]-/Pyrido[2,1-c]pyrrolo[1,2-a]quinoxalinones. Adv. Synth. Catal. 2010, 352, 1711-1717.

42. Waldmann, H.; Eberhardt, L.; Wittstein, K.; Kumar, K. Silver catalyzed cascade synthesis of alkaloid ring systems: Concise total synthesis of fascaplysin, homofascaplysin $\mathrm{C}$ and analogues. Chem. Commun. 2010, 46, 4622-4624.

Sample Availability: Samples of all target compounds are available from the authors.

(C) 2013 by the authors; licensee MDPI, Basel, Switzerland. This article is an open access article distributed under the terms and conditions of the Creative Commons Attribution license (http://creativecommons.org/licenses/by/3.0/). 